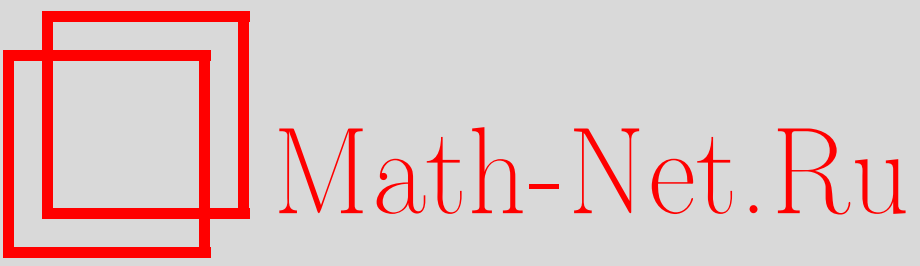

А. Милке, С. В. Зелик, Бесконечномерные траекторные аттракторы эллиптических краевых задач в цилиндрических областях, УМН, 2002, том 57, выпуск 4, 119-150

DOI: https://doi.org/10.4213/rm550

Использование Общероссийского математического портала Math-Net.Ru подразумевает, что вы прочитали и согласны с пользовательским соглашением

http: //www . mathnet.ru/rus/agreement

Параметры загрузки:

IP : 54.81 .137 .203

26 апреля 2023 г., 17:02:05 


\title{
БЕСКОНЕЧНОМЕРНЫЕ ТРАЕКТОРНЫЕ АТТРАКТОРЫ ЭЛЛИПТИЧЕСКИХ КРАЕВЫХ ЗАДАЧ В ЦИЛИНДРИЧЕСКИХ ОБЛАСТЯХ
}

\author{
А. Милке, С. ЗЕлик
}

\begin{abstract}
Работа посвящена изучению абстрактной модели нелинейной эллиптической краевой задачи второго порядка в цилиндрической области методами теории динамических систем. Показано, что при вьполнении некоторых естественных условий существенные решения рассматриваемой задачи описываются при помощи глобального аттрактора соответствующей траекторной динамической системы, которьй может иметь бесконечную фракталшную размерность и бесконечную топологическую энтропию. Кроме того, получены точные оценки сверху и снизу для колмогоровской $\varepsilon$-энтропии этих аттракторов.

Библиографиия: 42 названия.
\end{abstract}

\section{СОДЕРЖАНИЕ}

$\S 0$. Введение

$\S 1$. Априорные оценки. Существование решений

$\S 2$. Аттрактор

$\S 3$. Колмогоровская энтропия аттрактора и ее оценки сверху ........... 133

$\S 4$. Энтропия аттрактора: пример точной оценки снизу $\ldots . . \ldots \ldots \ldots . . . .139$

$\S 5$. Хаос в пространственных динамических системах ................ 143

Список литературы ................................................. 148

\section{$\S$ 0. Введение}

Пространственные динамические системы возникают при изучении нелинейных эллиптических краевых задач в цилиндрических областях, в которых пространственная координата, соответствуюшая оси цилиндра, играет роль времени. Использование теории динамических систем для исследования таких задач было инициировано работой [20], в которой было построено локальное центральное многообразие для квазилинейного эллиптического у равнения в полосе. Этот метод сведения эллиптической краевой задачи к динамической системе на пространственном центральном многообразии, позднее названньй редукцией Киржгеснера, получил дальнейшее развитие в работах [23], [24], [18], [17], [16], [5], [26], где он использовался для исследования различных задач математической физики, возникаюших, в частности, в гидродинамике и 
нелинейной теории упругости. Частный случай вариационных эллиптических задач исследовался в работе [25], в которой было доказано наличие гамильтоновой структуры у редуцированной динамической системы на пространственном центральном многообразии.

Методы, использующие качественную теорию динамических систем для исследования глобальной структуры множества ограниченных решений эллиптических краевых задач в цилиндрических областях, развивались параллельно, начиная с работ $[6],[27],[28]$. Основной идеей этих методов является введение вспомогательной эллиптической задачи в полуцилиндре $\Omega_{+}:=(0, \infty) \times \omega\left((t, x) \in \Omega_{+}\right)$с дополнительньм краевым условием $\left.u\right|_{t=0}=u_{0}$ на его основании и изучение оператора "эволюции"

$$
S_{t}: u(0, x) \rightarrow u(t, x),
$$

где $u(t, x)$ - ограниченное решение этой задачи, с динамической точки зрения. Тогда, как известно, если существует глобальньй аттрактор оператора (0.1), то он порождается сушественными решениями исходной краевой задачи во всем цилиндре $\Omega=\mathbb{R} \times \omega$, т.е. решениями, которые определены и ограничены в $\Omega$. Эта взаимосвязь позволяет изучать “динамику" сушественных решений, исследуя динамические свойства эволюционного оператора (0.1) на его аттракторе.

K сожалению, ограниченное решение вспомогательной задачи, описанной выше, как правило, не является единственным, поэтому полугрупа (0.1) может быть корректно определена только как полугруппа многозначных отображений. Появления многозначных отображений можно избежать, используя так назьваемьй траекторный подход, при котором множество $\mathscr{K}^{+}$всех ограниченных решений вспомогательной задачи, наделенное подходяшей топологией, рассматривается как (траекторное) фазовое пространство для динамической системы, порожденной полугруппй положительных сдвигов $\left(\mathscr{T}_{h}\right)_{h \geqslant 0}$ вдоль оси цилиндра, определенной по формуле

$$
\left(\mathscr{T}_{h} u\right)(t, x)=u(t+h, x) \text { для любых }(t, x) \in \Omega_{+}, \quad h \geqslant 0 .
$$

Если существует глобальный аттрактор этой полугрупшы, то он назьвается траекторным аттрактором исходной задачи. Если траекторньй аттрактор может быть вложен в конечномерное инвариантное многообразие, то это многообразие назьвается существенным многообразием, так как оно содержит все сушественные решения рассматриваемой задачи, см. [28], [36], [7], [33]. (Траекторньй подход, описанный вьше, используется также для изучения различных әволюиионных уравнений математической физики, для которых еше не решена проблема единственности решений, например, для трехмерной системы уравнений Навье-Стокса, нелинейных волновых уравнений с быстро растущими нелинейностями и др., см. [8].)

В случае эллиптических краевых задач второго порядка сушествует еще одна возможность избежать появления многозначных отображений, основанная на замене оператора (0.1) следуюшим эволюционным оператором:

$$
\mathbb{S}_{t}:\left(u(0), \partial_{t} u(0)\right) \rightarrow\left(u(t), \partial_{t} u(t)\right), \quad\left(u(0), \partial_{t} u(0)\right) \in \mathbb{K}^{+},
$$

где $\mathbb{K}^{+}$- множество всех "начальных данных" $\left(u(0), \partial_{t} u(0)\right)$, для которых вспомогательная задача имеет ограниченное решение. Тогда при некоторых дополнитель- 
ных ограничениях это решение будет единственным, и, следовательно, (0.3) корректно определяет непрерьвную полугруппу в фазовом пространстве $\mathbb{K}^{+}$(см. [6]). Заметим, однако, что эта полугруппа оказывается гомеоморфной полугруппе сдвигов $(0.2)$, определенной на траекторном фазовом пространстве $\mathscr{K}^{+}$(см., например, 22 ниже).

Альтернативньй подход, связанньй с непосредственным изучением “эволюционного" оператора (0.1), используя соответствуюшее обобшение понятия глобального аттрактора на случай полугрупп многозначных отображений, был предложен в работе [2].

Другие идеи и методы качественной теории динамических систем также применяются для исследования эллиптических краевых задач. Так, экспоненциальные дихотомии были построены в работе [32] для исследования бифуркаций уединенных волн. Теория Флоке в окрестности пространственно периодического решения была развита в работах [27], [12]. Индекс Конли был использован в [15] для доказательства сушествования нетривиальных гетероклинических решений. Описание структуры аттрактора в случае, когда вспомогательная задача имеет единственное решение, а исходная эллиптическая система допускает глобальную функцию Ляпунова, было получено в $[37]$.

Заметим, однако, что очень мало известно о хаусдорфовой и фрактальной размерности аттракторов эллиптических уравнений, несмотря на существование хорошо разработанной теории получения оценок этих размерностей в случае эволюционных уравнений (см., например, [34]). Фактически, к настояшему моменту известно только два весьма узких класса эллиптических краевых задач, аттрактор которых имеет конечную размерность. Первьй из них - это случай однозначной разрешимости вспомогательной задачи в полуцилиндре, при наличии которой эллиптическая задача в полуцилиндре сводится к некоторому эволюционному уравнению на его сечении (см. [37]), а второй - это случай сушествования сушественного многообразия, когда размерность аттрактора естественно мажорируется размерностью многообразия (см. $[28],[3])$.

В настоящей работе мы покажем, что размерность аттрактора может быть бесконечной для эллиптических краевых задач, не принадлежащих вьшеперечисленньм классам, и дадим количественное описание "толщины" таких аттракторов в терминах колмогоровской $\varepsilon$-энтропии.

Рассматривается следующая абстрактная квазилинейная эллиптическая задача:

$$
\left\{\begin{array}{l}
\ddot{u}-\gamma \dot{u}-A u=F(u, \dot{u}) \text { при } t>0, \\
\left.u\right|_{t=0}=u_{0} .
\end{array}\right.
$$

Здесь $u(t)$ - элемент некоторого гильбертова пространства $H$ со скалярным произведением $\langle\cdot, \cdot\rangle$. Линейньй оператор $A: D(A) \rightarrow H$ предполагается самосопряженным, положительно определенным $\left(\langle A u, u\rangle \geqslant \lambda_{0}\|u\|^{2}\right.$ для некоторого $\left.\lambda_{0} \geqslant 1\right)$ и имеющим компактньй обратньй (оператор $A^{-1}$ сушествует и компактен). Определим шкалу гильбертовых пространств $\left(H^{s}\right)_{s \in \mathbb{R}}$, порожденную оператором $A$, по формуле $H^{s}=$ $D\left(A^{s / 2}\right),\|\cdot\|_{s} \equiv\|\cdot\|_{H^{s}}=\left\|A^{s / 2} \cdot\right\|$. Введем также пространства $\mathbb{H}^{s}=H^{s} \times H^{s-1}$ с индуцированной на них естественной гильбертовой структурой.

Кроме того, предполагается, что $\gamma$ - ограниченньй симметрический оператор в $H$, а нелинейная функция $F$ удовлетворяет следуюшим условиям: сушествуют положительные константы $C$ и $\delta(\delta \ll 1)$ и монотонные функции $Q_{\mu}: \mathbb{R}^{+} \rightarrow \mathbb{R}^{+}$, определенные 
для любого $\mu>0$, такие, что

$$
\left\{\begin{array}{l}
\text { (a) } F \in C^{1}\left(H^{3 / 2-\delta} \times H^{1 / 2-\delta}, H\right), \\
\text { (b) } \mathrm{D}_{u} F(u, v) \geqslant-C-\frac{1}{2} A, \\
\text { (c) }\langle F(u, v), u\rangle \geqslant-C-\frac{1}{2}\left(\|u\|_{1}^{2}+\|v\|^{2}\right), \\
\text { (d) }\|F(u, v)\|^{2} \leqslant Q_{\mu}\left(\|u\|_{1 / 2}\right)+\mu\|u\|_{2}^{2}+C\left(\|u\|_{1}^{2}+\|v\|^{2}\right) .
\end{array}\right.
$$

Выбор конкретного вида уравнения (0.4) мотивирован следующей эллиптической краевой задачей в цилиндрической области $\Omega_{+}=\mathbb{R}^{+} \times \omega(\omega-$ ограниченная область в $\left.\mathbb{R}^{n}\right)$ :

$$
\left\{\begin{array}{l}
\ddot{u}-\gamma \dot{u}+\Delta_{x} u=f(u, \dot{u})+g(x) \text { при }(t, x) \in \Omega_{+}, \\
\left.u\right|_{\mathbb{R}^{+} \times \partial \omega}=0,\left.\quad u\right|_{t=0}=u_{0},
\end{array}\right.
$$

где $u=\left(u^{1}, \ldots, u^{k}\right) \in \mathbb{R}^{k}, \gamma=\gamma^{*} \in \mathscr{L}\left(\mathbb{R}^{k}, \mathbb{R}^{k}\right), g \in L^{2}(\omega)$, которая возникает, например, при изучении решений вида бегущей волны для соответствующего эволющионного уравнения в неограниченной цилиндрической области $\Omega=\mathbb{R} \times \omega$ (см., например, $[6],[2]$ или [36]).

Существование ограниченного решения $u(t), t \geqslant 0$, уравнения (0.4) для любого начального условия $u_{0} \in H^{3 / 2}$ доказано в $\S 1$. Кроме того, в этом параграфе мы вьведем диссипативную оценку для ограниченных решений $u \in W_{\mathrm{bd}}^{2}\left(\mathbb{R}^{+}\right)$(см. определение 1.1) уравнения (0.4), которая имеет фундаментальное значение для использования траекторного подхода, описанного вьше.

В $\S 2$ мы докажем, что абстрактное уравнение (0.4) обладает траекторным аттрактором $\mathscr{A}=\mathscr{A}^{\text {traj }}$, т.е. что полугруппа $(0.2)$, определенная в пространстве $\mathscr{K}^{+}$всех ограниченных решений $u \in W_{\mathrm{bd}}^{2}\left(\mathbb{R}^{+}\right)$, имеет глобальный аттрактор $\mathscr{A}$, которьй порождается сушественными решениями уравнения (0.4):

$$
\mathscr{A}^{\text {traj }}=\Pi_{+} \mathscr{K}
$$

где $\mathscr{K} \subset W_{\text {bd }}^{2}(\mathbb{R})$ - множество всех сушественных решений уравнения $(0.4)$, а $\Pi_{+}-$ оператор ограничения функции на полуось $\mathbb{R}^{+}$.

Кроме того, при вьполнении дополнительного условия

$$
\left\|\mathrm{D}_{u} F(u, v)\right\|_{H^{1} \rightarrow H}+\left\|\mathrm{D}_{v} F(u, v)\right\|_{H \rightarrow H} \leqslant Q\left(\|u\|_{3 / 2}+\|v\|_{1 / 2}\right)
$$

(где $Q$ - некоторая монотонная функция) на нелинейность $F$ мы докажем, что любое ограниченное решение $u(t)$ уравнения $(0.4)$ однозначно определяется парой начальных значений $\left(u(0), \partial_{t} u(0)\right)$, и проверим, таким образом, что полугруппа $(0.3)$ корректно определена в пространстве $\mathbb{K}^{+}$. Этот результат основьвается на оценках типа логарифмической вьпуклости для абстрактных эллиптических уравнений, см. [1], [6]. В этом параграфе мы также проверим, что естественная проекция $\Pi_{0}: \mathscr{K}^{+} \rightarrow \mathbb{K}^{+}$, определенная по формуле $\Pi_{0} u:=\left(u(0), \partial_{t} u(0)\right)$, является непрерьвным по Гёльдеру гомеоморфизмом и, следовательно, полугрупп (0.3) может быть определена по формуле

$$
\mathbb{S}_{h}:=\Pi_{0} \mathscr{T}_{h}\left(\Pi_{0}\right)^{-1} .
$$

Этот результат гарантирует существование глобального аттрактора $\mathbb{A} \subset \mathbb{K}^{+}$полугрупшы (0.3), а также доказьвает соотношение

$$
\mathbb{A}=\Pi_{0} \mathscr{A}^{\text {traj }} .
$$


В $\S 3$ мы используем понятие колмогоровской $\varepsilon$-энтропии для изучения количественных характеристик аттрактора $\mathscr{A}^{\text {traj }}$ для эллиптической задачи $(0.4)$, построенного в предыдущем параграфе. Детальное изложение колмогоровской энтропии дано, например, в работе [21], а ее приложения к эволюционным уравнениям математической физики рассмотрены в [9], [10], [38], [40], [11]. Основным результатом третьего параграфа является следуюшая оценка сверху для колмогоровской $\varepsilon$-энтропии $\mathbf{H}_{\varepsilon}\left(\left.\mathscr{A}^{\text {traj }}\right|_{(0, T)}\right)$ ограничений аттрактора $\mathscr{A}^{\text {traj }}$ на произвольньй конечньй интервал $(0, T)$ :

$$
\mathbf{H}_{\varepsilon}\left(\left.\mathscr{A}^{\mathrm{traj}}\right|_{(0, T)}\right) \leqslant C\left[T+\ln _{+} \frac{R_{0}}{\varepsilon}\right] \ln _{+} \frac{R_{0}}{\varepsilon}
$$

где $C$ и $R_{0}$ - положительные константы, не зависяшие от $\varepsilon>0$ и $T \geqslant 0$, a $\ln _{+} z:=$ $\max \{\ln z, 0\}$.

Заметим, что оценки (0.10) недостаточно, чтобы сделать вывод о конечности фрактальной размерности $\operatorname{dim}_{\text {fract }}(\mathbb{A})$ аттрактора. Более того, как показано в $\S 4$, эта величина, действительно, может быть бесконечной. В действительности, мы построили пример оператора $A$ и нелинейного отображения $F$, удовлетворяющего условиям $(0.5)$, такого, что колмогоровская энтропия соответствующего аттрактора допускает следующую оценку снизу:

$$
\mathbf{H}_{\varepsilon}\left(\left.\mathscr{A}^{\mathrm{traj}}\right|_{(0, T)}\right) \geqslant C^{\prime} T \ln _{+} \frac{R_{0}^{\prime}}{\varepsilon}
$$

для некоторых $C^{\prime}>0, R_{0}^{\prime}>0$, не зависящих от $T \geqslant 1$ и $\varepsilon>0$. Кроме того, используя рассуждения, основанные на логарифмической выпуклости, мы вьведем из (0.11) оценку

$$
\mathbf{H}_{\varepsilon}(\mathbb{A}) \geqslant C^{\prime \prime}\left(\ln _{+} \frac{R_{0}^{\prime \prime}}{\varepsilon}\right)^{3 / 2},
$$

которая показывает бесконечность следующих размерностей:

$$
\operatorname{dim}_{\text {fract }}\left(\left.\mathscr{A}^{\text {traj }}\right|_{(0, T)}\right)=\operatorname{dim}_{\text {fract }}(\mathbb{A})=\infty
$$

Наш пример базируется на контрпримере к теории Флоке для линейных эллиптических уравнений с периодическими коэффищиентами, построенном в [12]. Этот контрпример имеет вид

$$
\ddot{v}-A v=L_{1}(t) v+L_{2}(t) \dot{v}
$$

где $L_{1}$ и $L_{2}-$ периодичны по $t \in \mathbb{R}$ и подобраны так, чтобы существовало нетривиальное решение $v: \mathbb{R} \rightarrow H^{2}$, убьваюшее при $t \rightarrow \pm \infty$ быстрее любой экспоненты $\left(\|v(t)\|_{2} \leqslant c e^{-t^{2}}\right)$. Для построения этого контрпримера необходимо, чтобы

$$
L_{1} \in C_{\mathrm{per}}\left(\mathbb{R}, \mathscr{L}\left(H^{s+r_{1}}, H^{s}\right)\right) \text { и } L_{2} \in C_{\mathrm{per}}\left(\mathbb{R}, \mathscr{L}\left(H^{s+r_{2}}, H^{s}\right)\right)
$$

для некоторых $r_{1} \geqslant 1, r_{2} \geqslant 0$ и $s \geqslant 0$. С другой стороны, для нашей теоремы о существовании траекторного аттрактора (см. (0.5) и (0.7)) требуется, чтобы $r_{1} \leqslant 1$ и $r_{2} \leqslant 0$. Таким образом, наш пример находится в точности на границе области допустимых значений параметров $r_{1}$ и $r_{2}$. 
В $\S 5$ мы дадим более детальное исследование динамических свойств траекторной динамической системы, соответствуюшей примеру, построенному в предыдущем параграфе. В частности, мы покажем, что в отличие от динамических систем (ДС), порождаемых обькновенными дифференциальными уравнениями (ОДУ) и большинством естественных эволюционных уравнений в частных производных (УрЧП) в ограниченных областях, рассматриваемая ДС имеет бесконечную топологическую энтропию. Мы опишем хаотическую природу этой ДС при помоши гомеоморфного вложения схемы Бернулли с бесконечным числом символов. Отметим также, что этот тип хаотического поведения оказывается очень близок к поведению ДС, порождаемых эволюционными УрЧП в неограниченных областях, см. [39], [40].

Эллиптическая краевая задача (0.6) в полном цилиндре $\Omega=\mathbb{R} \times \omega$ может быть интерпретирована, в частности, как уравнение для нахождения положений равновесия соответствуюшей системы уравнений реакции-диффузии в $\Omega$ :

$$
\partial_{\eta} u=\ddot{u}-\gamma \dot{u}+\Delta_{x} u-f(u, \dot{u})-g(x),(t, x) \in \Omega, \eta>0,\left.u\right|_{\eta=0}=u^{0}
$$

где $t$ остается пространственной переменной, а роль физического времени играет переменная $\eta$. Известно (см. [4], [31], [14]), что при выполнении естественных условий на нелинейную функцию $f$ и внешнюю силу $g$ это уравнение обладает глобальным аттрактором $\mathscr{A}^{\text {glob }} \subset W_{\text {bd }}^{2}(\mathbb{R})$. Кроме того, очевидно, справедливо вложение

$$
\mathscr{K} \subset \mathscr{A}^{\text {glob }}
$$

где $\mathscr{K}$ - множество существенных решений эллиптической краевой задачи (0.6). Аналог оценок (0.10) и (0.11) для $\varepsilon$-энтропии глобального аттрактора $\mathscr{A}^{\text {glob }}$ был получен в работах [10], [38], [14], см. также $§ 5$ для более подробного обсуждения этой аналогии.

В следующей работе мы изучим вопрос о дополнительных условиях, гарантирующих конечность фрактальной размерности $\operatorname{dim}_{\text {fract }}(\mathbb{A})$ и/или топологической энтропии $h_{\mathrm{top}}\left(\mathbb{S}_{h}, \mathbb{A}\right)$. K настоящему времени этот факт известен только при наличии щели в спектре оператора $A$, гарантирующей сушествование сушественного многообразия [28], или при выполнении условия $\gamma \gg \mathrm{id}$, которое дает единственность решения задачи (0.4), см. [7], [37] и замечание 2.3 ниже.

Данная работа вьполнена при частичной поддержке Deutsche Forschungsgemeinschaft, DANSE, Mi 459/2-3. Авторы также выражают свою благодарность М. А. Ефендиеву, Д. В. Тураеву и М.И. Вишику за множество полезных обсуждений.

\section{§ 1. Априорные оценки. Существование решений}

В этом параграфе мы выведем несколько априорных оценок для решений задачи (0.4) и, основываясь на этих оценках, докажем существование ограниченных решений этой задачи. Для этого нам понадобятся следующие функциональные пространства.

ОПРЕДЕЛЕНИЕ 1.1. Для любых $-\infty \leqslant T_{1}<T_{2} \leqslant+\infty$ и любого $l \in \mathbb{R}^{+}$определим пространство

$$
W^{l}\left(T_{1}, T_{2}\right) \equiv L^{2}\left(\left(T_{1}, T_{2}\right), H^{l}\right) \cap W^{l, 2}\left(\left(T_{1}, T_{2}\right), H\right) .
$$

Для простоты мы будем писать далее $W^{l}(T)$ вместо $W^{l}(T, T+1)$. 
Обозначим через $W_{\text {loc }}^{l}\left(\mathbb{R}^{+}\right)$пространство Фреше, порождаемое полунормами $\|\cdot\|_{W^{l}(T)}, T \in \mathbb{R}^{+}$. Более того, определим также пространство

$$
W_{\mathrm{bd}}^{l}\left(\mathbb{R}^{+}\right) \equiv\left\{u \in W_{\mathrm{loc}}^{l}\left(\mathbb{R}^{+}\right):\|u\|_{l, b} \equiv \sup _{T \in \mathbb{R}^{+}}\|u\|_{W^{l}(T)}<\infty\right\} .
$$

Пространства $W_{\text {loc }}^{l}(\mathbb{R})$ и $W_{\text {bd }}^{l}(\mathbb{R})$ определяются аналогично, с заменой $T \in \mathbb{R}^{+}$на $T \in \mathbb{R}$.

Основньм результатом этого параграфа является следующая теорема.

ТЕОрема 1.2. Пусть выполнены условия (0.5). Тогда для любого $u_{0} \in H^{3 / 2}$ существует хотя бь одно решение $и \in W_{\mathrm{bd}}^{2}\left(\mathbb{R}^{+}\right)$задачи (0.4). Более того, существуют $C_{*}, \alpha>0$ и монотонная функция $Q: \mathbb{R}^{+} \rightarrow \mathbb{R}^{+}$такие, что любое решение $u \in W_{\mathrm{bd}}^{2}\left(\mathbb{R}^{+}\right)$задачи (0.4) удовлетворяет оченке

$$
\|u\|_{W_{\mathrm{bd}}^{2}(T)} \leqslant Q\left(\left\|u_{0}\right\|_{3 / 2}\right) e^{-\alpha T}+C_{*} \quad \text { npu } \quad T \geqslant 0 .
$$

ДоказАТЕльство. Мы выведем сначала априорную оценку (1.3). Сушествование решения мы докажем позднее, основываясь на этой оценке.

Следуя [37], умножим уравнение (0.4) скалярно в $H$ на функцию $\rho(t) u(t)$ и проинтегрируем по $t \in[\tau,+\infty)$. Здесь $\rho: \mathbb{R}^{+} \rightarrow(0, \infty)$ - весовая функция такая, что $\int_{0}^{\infty} \rho(t) d t<\infty$ и $|\dot{\rho}(t)| \leqslant \varepsilon \rho(t)$ для любых $t \geqslant 0$. Интегрируя полученное равенство по частям и используя симметричность $\gamma$, получим

$$
\begin{aligned}
\int_{\tau}^{\infty} & {\left[\|\dot{u}\|^{2}+\|u\|_{1}^{2}\right] \rho d t+\left[\frac{\rho}{2} \partial_{t}\|u\|^{2}-\frac{\rho}{2}\langle\gamma u, u\rangle\right]_{t=\tau} } \\
& =\int_{\tau}^{\infty}[-\langle u, F(u, \dot{u})\rangle \rho-\langle u, \dot{u}\rangle \dot{\rho}+\langle\gamma u, u\rangle \dot{\rho} / 2] d t \\
& \leqslant \int_{\tau}^{\infty}\left[C+\frac{1}{2}\left(\|u\|_{1}^{2}+\|\dot{u}\|^{2}\right)+\varepsilon\|u\|\|\dot{u}\|+\varepsilon\|\gamma\|\|u\|^{2}\right] \rho d t
\end{aligned}
$$

Здесь мы использовали также условие $(0.5)(\mathrm{c})$. Взяв $\rho(t):=e^{\varepsilon(t-\tau)}$ и зафиксировав достаточно малое $\varepsilon>0$, получим

$$
\frac{d}{d \tau}\|u(\tau)\|^{2} \leqslant C^{\prime}+C^{\prime}\|u(\tau)\|^{2} \text { при } \tau \geqslant 0 .
$$

Из неравенства Гронуолла следует теперь первая априорная оценка для $H$-нормы решения

$$
\|u(t)\|^{2} \leqslant e^{C^{\prime} t}\left\|u_{0}\right\|^{2}+e^{C^{\prime} t}-1 \text { при } t \geqslant 0 .
$$

Естественно, эта оценка полезна только при малых $t$, так как ее правая часть растет экспоненщиально при $t \rightarrow \infty$.

В качестве следуюшего шага мы докажем аналог оценки (1.3) для $W^{1}(T)$-нормы решения, в которой правая часть не будет расти при $t \rightarrow \infty$. Для этого необходимо уделить особое внимание начальному условию $u_{0}=\left.u\right|_{t=0}$. Действительно, согласно абстрактной теореме о следах, сушествует линейньй ограниченный оператор $\mathbb{T}: H^{3 / 2} \rightarrow W^{2}\left(\mathbb{R}^{+}\right)$такой, что $v=\mathbb{T} u_{0}$ удовлетворяет условиям

$$
v(0)=u_{0}, \quad \operatorname{supp} v \subset[0,1] \text { и }\|v\|_{W^{2}(0)} \leqslant C\left\|u_{0}\right\|_{3 / 2} .
$$


Переписав уравнение (0.4) относительно новой неизвестной $w=u-v$, будем иметь

$$
\left\{\begin{array}{l}
\ddot{w}-\gamma \dot{w}-A w=F(w+v, \dot{w}+\dot{v})-h(t), \\
\left.w\right|_{t=0}=0
\end{array}\right.
$$

где $h=\ddot{v}-\gamma \dot{v}-A v$. Следовательно,

$$
\operatorname{supp} h \in[0,1], \quad\|h\|_{L^{2}(0)} \leqslant C_{1}\left\|u_{0}\right\|_{3 / 2} .
$$

Взяв скалярное произведение $(1.8)$ в $H$ с $\rho(t) w(t)$, проинтегрировав по $t \in \mathbb{R}^{+}$и использовав краевое условие $w(0)=0$, рассуждая так же, как и при вьводе (1.4), получим

$$
\begin{aligned}
& \int_{0}^{\infty} {\left[\|\dot{w}\|^{2}+\|w\|_{1}^{2}\right] \rho d t } \\
& \quad=\int_{0}^{\infty}\left[-\langle w, F(v+w, \dot{v}+\dot{w})\rangle-\langle w, h\rangle+\frac{\dot{\rho}}{2 \rho}[\langle\gamma w, w\rangle-2\langle w, \dot{w}\rangle]\right] \rho d t
\end{aligned}
$$

Наиболее сложным для оценки является первое слагаемое в правой части (1.10), содержашее нелинейность $F$. Для его оценки мы напомним, что функции $v(t)$ и $h$ отличны от нуля только при $t \in[0,1]$. Поэтому при $t \in[0,1]$ мы должны использовать более слабые оценки $(0.5)(\mathrm{b})+(\mathrm{d})$ нелинейности, тогда как при $t \geqslant 1$ мы можем использовать более сильную оценку $(0.5)(\mathrm{c})$. Таким образом, для ненулевого $v$ получим

$$
\begin{aligned}
-\langle w, F(v+w, \dot{v}+\dot{w})\rangle= & -\langle w, F(v+w, \dot{v}+\dot{w})-F(v, \dot{v}+\dot{w})\rangle-\langle w, F(v, \dot{v}+\dot{w})\rangle \\
\leqslant & C\|w\|^{2}+\frac{1}{2}\|w\|_{1}^{2}+\frac{1}{4 \alpha}\|w\|^{2}+\alpha\|F(v, \dot{v}+\dot{w})\|^{2} \\
\leqslant & C(\alpha)\|w\|^{2}+\frac{1}{2}\|w\|_{1}^{2}+\alpha Q_{\mu}\left(\|v\|_{1 / 2}\right) \\
& +\alpha \mu\|v\|_{2}^{2}+\alpha C\left(\|v\|_{1}^{2}+\|\dot{v}+\dot{w}\|^{2}\right) \\
\leqslant & C(\alpha)\|w\|^{2}+\frac{1}{2}\|w\|_{1}^{2}+Q_{1}(\alpha)\left(\left\|u_{0}\right\|_{3 / 2}\right)+2 \alpha C\|\dot{w}\|^{2} .
\end{aligned}
$$

При $t \geqslant 1$ мы используем лучшую оценку $(0.5)($ c), так как в этом случае $v \equiv 0$ :

$$
-\langle w, F(v+w, \dot{v}+\dot{w})\rangle=-\langle F(w, \dot{w}), w\rangle \leqslant C_{2}+\frac{1}{2}\left(\|w\|_{1}^{2}+\|\dot{w}\|^{2}\right) .
$$

Подставив эти оценки в $(1.10)$, зафиксировав достаточно малые константы $\alpha$ и $\varepsilon:=\sup |\dot{\rho}| / \rho$ и использовав неравенство (1.6) для оценки выражения $C(\alpha)\|w(t)\|^{2}$ при $t \in[0,1]$, мы вьведем следуюшую оценку:

$$
\int_{0}^{\infty}\left[\|\dot{w}\|^{2}+\|w\|_{1}^{2}\right] \rho d t \leqslant \int_{0}^{1} Q_{2}\left(\left\|u_{0}\right\|_{3 / 2}\right) \rho d t+\int_{0}^{\infty}\left[C_{2}+\frac{3}{4}\left[\|\dot{w}\|^{2}+\|w\|_{1}^{2}\right]\right] \rho d t .
$$

Фиксировав в этом неравенстве весовую функцию $\rho(t):=e^{-\varepsilon|t-T|}, T \geqslant 0$, получим

$$
\begin{aligned}
\|w\|_{W^{1}(T)}^{2} & =\int_{T}^{T+1}\left[\|\dot{w}\|^{2}+\|w\|_{1}^{2}\right] d t \leqslant e^{\varepsilon} \int_{0}^{\infty}\left[\|\dot{w}\|^{2}+\|w\|_{1}^{2}\right] e^{-\varepsilon|t-T|} d t \\
& \leqslant 4 e^{\varepsilon} \sup _{t \in[0,1]} e^{-\varepsilon|t-T|} Q_{2}\left(\left\|u_{0}\right\|_{3 / 2}\right)+e^{\varepsilon} \frac{8}{\varepsilon} C_{2} \leqslant 5 Q_{2}\left(\left\|u_{0}\right\|_{3 / 2}\right) e^{-\varepsilon T}+\frac{10 C_{2}}{\varepsilon} .
\end{aligned}
$$


Возвращаясь обратно к переменной $u=v+w$ и используя неравенство (1.7) для функции $v$, мы докажем следуюшую оценку:

$$
\|u\|_{W^{1}(T)} \leqslant Q_{3}\left(\left\|u_{0}\right\|_{3 / 2}\right) e^{-\varepsilon T}+C_{3} .
$$

Теперь мы готовы завершить доказательство априорной оценки решения в пространстве $W^{2}(T)$. Для этого мы используем регулярность решений уравнения $\ddot{z}-A z=f$ с условиями Дирихле на границе, которое является абстрактной эллиптической краевой задачей. Рассмотрим интервал $J_{T}=(\max \{0, T-1\}, T+1)$ и введем срезающую функцию $\psi: \mathbb{R} \rightarrow[0,1]$ такую, что $\psi(t)=1$ при $t \in[0,1]$ и $\psi(t)=0$ при $t \notin[-1,2]$. Пусть также $\psi_{T}(t)=\psi(t-T)$ и $w_{T}=\psi_{T} w$. Тогда, согласно $(1.8)$, последняя функция удовлетворяет уравнению

$$
\left\{\begin{array}{l}
\ddot{w}_{T}-A w_{T}=f_{T} \equiv \psi_{T}(\gamma \dot{w}+F(u, \dot{u})+h)+2 \dot{\psi}_{T} \dot{w}+\ddot{\psi}_{T} w \text { при } t \in J_{T}, \\
w_{T}=0 \text { при } t \in \partial J_{T} .
\end{array}\right.
$$

Применив теорему о регулярности решений к линейному эллиптическому уравнению (1.12) и использовав условия (0.5), получим

$$
\begin{aligned}
\left\|w_{T}\right\|_{W^{2}\left(J_{T}\right)}^{2} & \leqslant C\left[\|w\|_{W^{1}\left(J_{T}\right)}^{2}+\|h\|_{L^{2}\left(J_{T}\right)}^{2}+\left\|\psi_{T} F(u, \dot{u})\right\|_{L^{2}\left(J_{T}\right)}^{2}\right] \\
& \leqslant Q_{4}\left(\left\|u_{0}\right\|_{3 / 2}\right) e^{-\varepsilon T}+C_{4}+\int_{J_{T}} C\left[Q_{\mu}\left(\|u(t)\|_{1 / 2}\right)+\mu \psi_{T}(t)^{2}\|w(t)\|_{2}^{2}\right] d t \\
& \leqslant Q_{5}\left(\left\|u_{0}\right\|_{3 / 2}\right) e^{-\varepsilon T}+C_{5}+C \mu\left\|w_{T}\right\|_{W^{2}\left(J_{T}\right)}^{2} .
\end{aligned}
$$

Здесь мы использовали условие $(0.5)(\mathrm{d})$ и оценку (1.11). Выбрав константу $\mu$ в оценке (1.13) достаточно малой, будем иметь

$$
\|w\|_{W^{2}(T)} \leqslant C\left\|w_{T}\right\|_{W^{2}\left(J_{T}\right)} \leqslant 2 Q_{5}\left(\left\|u_{0}\right\|_{3 / 2}\right) e^{-\varepsilon T}+2 C_{5} .
$$

Таким образом, априорная оценка (1.3) доказана, и для завершения доказательства теоремы 1.2 остается проверить сушествование решения $u \in W_{\mathrm{bd}}^{2}\left(\mathbb{R}^{+}\right)$рассматриваемой задачи. Для этого мы сначала построим для любого $N \in \mathbb{N}$ решение $u_{N}(t)$ следующей вспомогательной задачи вида (0.4) на конечном интервале:

$$
\left\{\begin{array}{l}
\ddot{u}_{N}-\gamma \dot{u}_{N}-A u=F\left(u_{N}, \dot{u}_{N}\right) \text { при } t \in(0, N) \\
\left.u_{N}\right|_{t=0}=u_{0},\left.\quad u_{N}\right|_{t=N}=0
\end{array}\right.
$$

а затем получим решение исходной задачи предельным переходом $N \rightarrow \infty$.

Повторяя дословно вывод оценки (1.3), мы получим, что любое решение $u_{N} \in$ $W^{2}(0, T)$ задачи $(1.15)$ удовлетворяет оценке

$$
\left\|u_{N}\right\|_{W^{2}(T)} \leqslant Q\left(\left\|u_{0}\right\|_{H^{3 / 2}}\right) e^{-\alpha T}+C_{*} \text { при } T \in[0, N-1],
$$

в которой функция $Q$ и константы $C_{*}$ и $\alpha$ такие же, как и в оценке $(1.3)$, и, следовательно, не зависят от $N \in \mathbb{N}$. Заметим также, что, согласно $(0.5)($ а) и абстрактной теореме о следах, нелинейный оператор $F: u \rightarrow F(u, \dot{u})$ является компактным и непрерьвньм отображением из $W^{2}(0, N)$ в $W^{0}(0, N)$ для любого конечного $N$. Таким образом, сушествование решения $u_{N}$ задачи (1.15) может быть выведено стандартньм образом из 
априорной оценки (1.16) при помоши теоремы Лере-Шаудера о неподвижной точке, см., например, [36], [37].

Для построения решения $u$ исходной задачи (0.4) мы заметим, что, согласно (1.16), последовательность $u_{N}$ равномерно ограничена в $W^{2}(0, T)$ при любом $T>0$. Поэтому, благодаря рефлексивности пространства $W^{2}(0, T)$ и канторовской диагональной процедуре, мы можем без ограничения общности считать, что $u_{N}$ слабо сходятся в пространстве $W_{\text {loc }}^{2}\left(\mathbb{R}_{+}\right)$к некоторой функции $u \in W_{\mathrm{bd}}^{2}\left(\mathbb{R}^{+}\right)$. Переходя к пределу $N \rightarrow \infty$ в уравнениях (1.15), мы получим, что $u$ - решение уравнения $(0.4)$. Действительно, предельньй переход в линейных членах уравнения (1.15) очевиден, и нелинейное слагаемое также не вызывает дополнительных трудностей, благодаря условию $(0.5)(\mathrm{a})$ и наличию сильной сходимости

$$
\left(u_{N}, \dot{u}_{N}\right) \rightarrow(u, \dot{u}) \text { в пространстве } C_{\mathrm{loc}}\left(H^{3 / 2-\delta} \times H^{1 / 2-\delta}\right)
$$

для любого $\delta>0$. Теорема 1.2 доказана.

\section{§2. Аттрактор}

Этот параграфф посвящен исследованию поведения решений задачи $(0.4)$ при $t \rightarrow \infty$. Заметим, что условия (0.5) гарантируют лиш существование решения $u$, но не его единственность, следовательно, мы не можем построить ДС, соответствующую (0.4), стандартным образом. Для того чтобы обойти эту трудность, мы будем использовать траекторный подход, развитый в работе [8] для случая эволюционных задач без единственности и в работе [36] для случая эллиптических краевых задач.

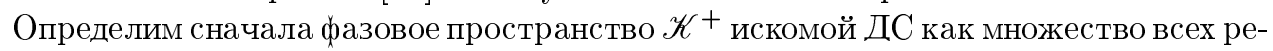
шений задачи $(0.4)$, определенных при $\mathbb{R}^{+}$и ограниченных при $t \rightarrow \infty$, т.е.

$$
\mathscr{K}^{+} \equiv\left\{u \in W_{\mathrm{bd}}^{2}\left(\mathbb{R}^{+}\right): u \text { - решение }(0.4) \text { для некоторого } u_{0} \in H^{3 / 2}\right\} .
$$

Так как наше уравнение не зависит явно от $t$, то полугрупша $\left(\mathscr{T}_{h}\right)_{h} \geqslant 0$ сдвигов вдоль оси $t$ действует в $\mathscr{K}^{+}$:

$$
\mathscr{T}_{h}: \mathscr{K}^{+} \rightarrow \mathscr{K}^{+}, \quad\left(\mathscr{T}_{h} u\right)(t) \equiv u(t+h), \quad h \geqslant 0 .
$$

Наделим множество $\mathscr{K}^{+}$локальной топологией, индуцированной вложением $\mathscr{K}^{+}{ }_{\text {в }}$ пространство $\Phi$ реше $W_{\text {loc }}^{2}\left(\mathbb{R}^{+}\right)$. Так как $W_{\text {loc }}^{2}\left(\mathbb{R}^{+}\right)$метризуемо, то $\mathscr{K}^{+}$также является метризуемым топологическим пространством.

ОПРЕДЕЛЕНИЕ 2.1. Множество $\mathscr{K}^{+}$, наделенное локальной топологией, назьвается траекторнылм фазовым пространством задачи $(0.4)$; полугруппа $\left(\mathscr{T}_{h}\right)_{h \geqslant 0}$, определенная в $(2.1)$, называется траекторной ДC, порожденной задачей $(0.4)$; глобальньй аттрактор $\mathscr{A}_{\text {полугруппы }}\left(\mathscr{T}_{h}\right)_{h \geqslant 0}$, действующей в $\mathscr{K}^{+}$, назьвается траекторным аттрактором задачи (0.4) и обозначается символом $\mathscr{A}^{\text {traj }}$.

ЗАмЕчАниЕ 2.2. Напомним, что по определению глобальньй аттрактор $\mathscr{A}$ полугрупшы $\mathscr{T}_{h}$ в $\mathscr{K}^{+}$должен притягивать ограниченные подмножества $\mathscr{K}^{+}$, хотя ограниченность является метрическим понятием и априори может зависеть от выбора метрики в пространстве $W_{\text {loc }}^{2}\left(\mathbb{R}^{+}\right)$. Однако как нетрудно показать, используя оценку $(1.3)$, в нашем случае множество $B \subset \mathscr{K}^{+}$ограничено в $W_{\text {lос }}^{2}(\mathbb{R})$ тогда и только тогда, когда $B$ ограничено в $W_{\mathrm{bd}}^{2}\left(\mathbb{R}^{+}\right)$, и, следовательно, “ограниченные" подмножества $\mathscr{K}^{+}$определены корректно. 


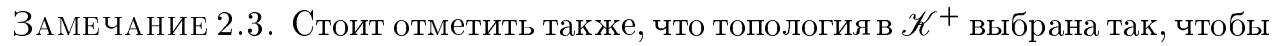
в случае, когда задача (0.4) имеет единственное решение, которое непрерьвно зависит от начальных условий $u_{0}$ (см. [7], [37] по поводу достаточных условий), полугруппа $\mathscr{T}_{h}$ совпадала бы с точностью до гомеоморфизма (и даже с точностью до $C^{1}$-диффеоморфизма в предположениях работы [37]) с “обычной” полугруппой $S_{h}: H^{3 / 2} \rightarrow H^{3 / 2}$, $S_{h} u_{0}=u(h)$.

Для формулировки следующей теоремы необходимо ввести понятие существенного решения. По определению, это решение задачи (0.4), определенное для всех $t \in \mathbb{R}$ и принадлежащее пространству $W_{\text {bd }}^{2}(\mathbb{R})$. Обозначим также через $\mathscr{K}, \mathscr{K} \subset W_{\text {bd }}^{2}(\mathbb{R})$, существенное множсество уравнения (0.4), которое состоит из всех существенных решений (см. [28]).

ТЕОРЕМА 2.4. При выполнении сформулированных выше условий уравнение (0.4) обладает траекторным аттрактором $\mathscr{A}=\mathscr{A}^{\text {traj }}$, который допускает следующее описание:

$$
\mathscr{A}=\Pi_{+} \mathscr{K}
$$

где $\mathscr{K} \subset W_{\mathrm{bd}}^{2}(\mathbb{R})$ - существенное множсество уравнения $(0.4)$, а $\Pi_{+}-$оператор ограничения функиии на полуось $\mathbb{R}^{+}$.

ДокАЗАТЕльство. Согласно теореме о сушествовании аттрактора для абстрактных полугрупп (см., например, [4]) достаточно проверить, что

(i) множество $\mathscr{K}^{+}$является полным метрическим пространством;

(ii) оператор $\mathscr{T}_{h}: \mathscr{K}^{+} \rightarrow \mathscr{K}^{+}$непрерьвен при фиксированном $h$;

(iii) полугруппа $\mathscr{T}_{h}$ обладает предкомпактным поглощающим множеством $B_{0}$ в $\mathscr{K}^{+}$, т.е. таким, что для любого ограниченного $B \subset \mathscr{K}^{+}$существует $\tau=\tau(B)$ такое, что $\mathscr{T}_{h} B \subset B_{0}$ при $h \geqslant \tau$.

Проверим эти условия. Действительно, так как пространство $W_{\text {loc }}^{2}\left(\mathbb{R}^{+}\right)$является полным, то первое условие следует из замкнутости $\mathscr{K}^{+}{ }_{\text {в }} W_{\text {loc }}^{2}\left(\mathbb{R}^{+}\right)$. Последний факт очевиден, так как предел решений уравнения (0.4) также является решением с соответствуюшим начальным условием $u_{0}$ (см. конец доказательства теоремы 1.2). Непрерьвность оператора $\mathscr{T}_{h}$ также очевидна, так как $\mathscr{T}_{h}-$ сдвиг вдоль оси $t$.

Таким образом, остается построить предкомпактное поглощающее множество $B_{0} \subset \mathscr{K}^{+}$. Из оценки (1.3) следует, что

$$
B_{*}=\left\{u \in W_{\mathrm{loc}}^{2}\left(\mathbb{R}^{+}\right):\|u\|_{W_{\mathrm{bd}}^{2}\left(\mathbb{R}^{+}\right)} \leqslant 2 C_{*}\right\} \cap \mathscr{K}^{+} \neq \varnothing
$$

является поглошаюшим множеством $\mathscr{T}_{h}$ в $\mathscr{K}^{+}$. Следовательно, $B_{0}=\mathscr{T}_{1} B_{*}-$ также поглощающее множество полугруппы $\mathscr{T}_{h}$. Проверим, что это множество - предкомпакт в $W_{\text {loc }}^{2}\left(\mathbb{R}^{+}\right)$. Для этой цели мы воспользуемся регулярностью решений эллиптических уравнений.

Согласно канторовской диагональной процедуре, достаточно доказать, что для любого $T \geqslant 1$ множество $\left.B_{*}\right|_{[T, T+1]}=\left\{\left.u\right|_{[T, T+1]}: u \in B_{*}\right\}$ предкомпактно в $W^{2}(T)$. Пусть $\left(u_{n}\right)_{n \in \mathbb{N}}-$ произвольная последовательность в $B_{*}$. Определим функции $z_{T, n}=$ 
$\psi_{T} u_{n}$, где срезаюшая функция $\psi_{T}$ такая же, как и в формуле (1.12). Тогда функция $z_{T, n}$ - решение уравнения

$$
\left\{\begin{array}{l}
\ddot{z}_{T, n}-A z_{T, n}=h_{T, n} \equiv \psi_{T}\left(\gamma \dot{u}_{n}+F\left(u_{n}, \dot{u}_{n}\right)\right)+2 \dot{\psi}_{T} \dot{u}_{n}+\ddot{\psi}_{T} u_{n}, \\
z_{T, n}(T-1)=z_{T, n}(T+2)=0 .
\end{array}\right.
$$

Так как последовательность $\left(u_{n}\right)_{n \in \mathbb{N}}$ ограничена в $W_{\mathrm{bd}}^{2}\left(\mathbb{R}^{+}\right)$, то из компактности вложения $W^{2}((T-1, T+2)) \subset W^{2-\delta}((T-1, T+2))$ и условия $(0.5)($ а) следует, что (после перехода к подпоследовательности, если это необходимо) $h_{T, n} \rightarrow h_{T}$ в пространстве $L^{2}((T-1, T+2))$. Применив теперь теорему о регулярности к уравнению $(2.4)$, получим, что $z_{T, n}=\psi_{T} u_{n} \rightarrow u_{T}$ в $W^{2}((T-1, T+2))$. Так как $\psi_{T}(t)=1$ при $t \in[T, T+1]$, то отсюда следует, что $u_{n} \rightarrow u$ в $W^{2}(T)$. Теорема 2.4 доказана.

Во второй части этого параграфа мы дадим другую интерпретацию ДС, порожденной уравнением (0.4), которая проясняет природу неединственности решений задачи (0.4). Для этой цели нам понадобится дополнительное условие (0.7) на нелинейность $F$ и некоторые оценки на разность $v(t):=u_{1}(t)-u_{2}(t)$ между произвольными решениями $u_{1}, u_{2} \in W_{\mathrm{bd}}^{2}\left(\mathbb{R}^{+}\right)$уравнения $(0.4)$, которая удовлетворяет следуюшему абстрактному линейному эллиптическому уравнению:

$$
\ddot{v}-A v=L_{1}(t) v+L_{2}(t) \dot{v},
$$

где операторы $L_{j}$ задаются формулами:

$$
\begin{aligned}
& L_{1}(t):=\int_{0}^{1} \mathrm{D}_{u} F\left(u_{1}(t)+s v(t), \dot{u}_{1}(t)+s \dot{v}(t)\right) d s, \\
& L_{2}(t):=\gamma+\int_{0}^{1} \mathrm{D}_{v} F\left(u_{1}(t)+s v(t), \dot{u}_{1}(t)+s \dot{v}(t)\right) d s .
\end{aligned}
$$

Более того, из условий (0.7) и теоремы 1.2 следует, что

$$
\left\|L_{1}(t)\right\|_{H^{1} \rightarrow H}+\left\|L_{2}(t)\right\|_{H \rightarrow H} \leqslant M, \quad t \in \mathbb{R}^{+},
$$

где константа $M$ зависит только от $\left\|u_{1}(0)\right\|_{H^{3 / 2}}$ и $\left\|u_{2}(0)\right\|_{H^{3 / 2}}$ и, следовательно, равномерно ограничена на ограниченных подмножествах $\mathscr{K}^{+}$.

Для решений уравнения (2.5) справедлива следующая теорема.

Теорема 2.5. Пусть $J=(0, T) u v \in W^{2}(J)$ удовлетворяет (2.5). Предположим, что существует $M>0$ такое, что

$$
\sup _{t \in J}\left\|L_{1}(t)\right\|_{H^{1} \rightarrow H} \leqslant M, \quad \sup _{t \in J}\left\|L_{2}(t)\right\|_{H \rightarrow H} \leqslant M .
$$

(Напомним, что $\left.\left\|L_{1}\right\|_{H^{1} \rightarrow H}=\left\|L A^{-1 / 2}\right\|_{H \rightarrow H}.\right)$ Пусть $y(t):=\|v(t)\|_{1}^{2}+\|\dot{v}(t)\|^{2}$. Тогда $y: J \rightarrow \mathbb{R}$ удовлетворяет следующим двум оченкам, справедливьм для любых $t \in J:$

$$
y(t) \geqslant y(0) e^{-2 M^{2} t^{2}-b t}, \quad \text { əдe } \quad b=4 M-4\left\langle A^{3 / 4} v(0), A^{1 / 4} \dot{v}(0)\right\rangle / y(0),
$$

$$
y(t) \leqslant[y(0)]^{1-t / T}[y(T)]^{t / T} e^{2 M(M+4 / T) t(T-t)} .
$$

Наличие вложения $W^{2}((0, T)) \rightarrow C\left([0, T], H^{3 / 2}\right) \cap C^{1}\left([0, T], H^{1 / 2}\right)$ гарантирует, что константа $b$ в (2.8) определена корректно. 
ДокАЗАТЕльство. Доказательство этой теоремы основано на оценках типа логарифмической вьпуклости, полученных в работе [1]. Действительно, введем функцию

$$
\xi(t)=\left(\begin{array}{c}
\dot{v}(t)+A^{1 / 2} v(t) \\
\dot{v}(t)-A^{1 / 2} v(t)
\end{array}\right), \quad \xi \in W_{\mathrm{bd}}^{1}(J)^{2} \subset C_{\mathrm{bd}}\left(J, H^{1 / 2} \times H^{1 / 2}\right) .
$$

Тогда из (2.5) следует, что $\xi$ - решение линейного уравнения

$$
\dot{\xi}(t)-\mathscr{B} \xi(t)=\mathscr{C}(t) \xi(t), \text { где } \mathscr{B}=\left(\begin{array}{cc}
-A^{1 / 2} & 0 \\
0 & A^{1 / 2}
\end{array}\right), \mathscr{C}(t)=\frac{1}{2}\left(\begin{array}{ll}
\mathscr{C}_{1}(t) & \mathscr{C}_{2}(t) \\
\mathscr{C}_{1}(t) & \mathscr{C}_{2}(t)
\end{array}\right),
$$

$\mathscr{C}_{1}=L_{2}+L_{1} A^{-1 / 2}$ и $\mathscr{C}_{2}=L_{2}-L_{1} A^{-1 / 2}$. Элементарные вычисления показьвают, что $2 y(t)=\|\xi(t)\|_{*}^{2} \equiv\left\|\xi_{1}(t)\right\|^{2}+\left\|\xi_{2}(t)\right\|^{2}$. Более того, из условия (2.7) следует, что оператор $\mathscr{C}(t)$ удовлетворяет оценке

$$
\|\mathscr{C}(t) \xi\|_{*} \leqslant 2 M\|\xi\|_{*} \text { для любых } t \in J .
$$

Предположим, что $y(\tau)>0$ для некоторого $\tau \in J$ (в противном случае доказывать нечего). Тогда из (2.10) следует, что сушествует максимальньй относительно открытьй интервал $J_{\tau}$ в $[0, T]$ такой, что $\tau \in J_{\tau}$ и $y(t)>0$ при $t \in J_{\tau}$. Определим функцию $\alpha: J_{\tau} \rightarrow \mathbb{R}$ по формуле

$$
\alpha(t)=\ln y(t)-\int_{\tau}^{t} \Phi(s) d s, \quad \text { где } \Phi(t)=\frac{\langle\mathscr{C}(t) \xi(t), \xi(t)\rangle_{*}}{y(t)},
$$

$\langle\cdot, \cdot\rangle_{*}-$ скалярное произведение в $H \times H$.

Продифференцировав (2.13) по $t$, использовав определение функции $\Phi(t)$ и уравнение (2.11), после несложных вычислений получим

$$
\dot{\alpha}=\langle\mathscr{B} \xi, \xi\rangle_{*} / y, \quad \ddot{\alpha}=\frac{2}{y}\left[\langle\eta, \eta\rangle_{*}+\langle\eta, \mathscr{C} \xi\rangle_{*}\right], \quad \text { где } \quad \eta=\mathscr{B} \xi-\frac{\langle\mathscr{B} \xi, \xi\rangle_{*}}{2 y} \xi .
$$

Из неравенства Коши-Шварца и оценки (2.12) следует теперь, что

$$
\ddot{\alpha}(t) \geqslant-\frac{\|\mathscr{C}(t) \xi(t)\|_{*}^{2}}{2 y(t)} \geqslant-4 M^{2} \text { при } t \in J_{\tau} .
$$

Эта оценка показьвает, что функция $J_{\tau} \ni t \mapsto \widetilde{\alpha}(t)=\alpha(t)+2 M^{2} t^{2}$ является выпуклой. Эта вьпуклость, вместе с предположением $y(\tau)>0$, немедленно показьвает, что интервал $J_{\tau}$ совпадает с $J=[0, T]$.

Выбрав $\tau=0$ в определении (2.13) и пропотенцировав неравенство $\widetilde{\alpha}(t) \geqslant \widetilde{\alpha}(0)+$ $\dot{\tilde{\alpha}}(0) t$, будем иметь

$$
y(t) \geqslant y(0) e^{-2 M^{2} t^{2}+\dot{\alpha}(0) t+\int_{0}^{t} \Phi(s) d s} .
$$

Это неравенство, вместе с очевидными оценками $|\Phi(s)| \leqslant 4 M$ и $\dot{\alpha}(0) \geqslant-b$, доказывает оценку (2.8). Оценка (2.9) вьводится аналогичньм образом из неравенства $\widetilde{\alpha}(t) \leqslant(1-t / T) \widetilde{\alpha}(0)+(t / T) \widetilde{\alpha}(T)$. Теорема 2.5 доказана. 
Напомним теперь, что $\mathbb{H}^{s}=H^{s} \times H^{s-1}$, введем линейное отображение следа по формуле

$$
\Pi_{0}: W_{\mathrm{loc}}^{2}\left(\mathbb{R}^{+}\right) \rightarrow \mathbb{H}^{3 / 2}, \quad \Pi_{0} u=(u(0), \dot{u}(0)),
$$

и определим множество $\mathbb{K}^{+}=\Pi_{0} \mathscr{K}^{+} \subset \mathbb{H}^{3 / 2}$. Очевидно, что множество $\mathbb{K}^{+}$замкнуто и, согласно теореме 2.5, отображение $\left.\Pi_{0}\right|_{\mathscr{K}^{+}}: \mathscr{K}^{+} \rightarrow \mathbb{K}^{+}$является биекцией. Это означает, что при выполнении условий теоремы 2.5 задача Коши

$$
\left\{\begin{array}{l}
\ddot{u}-\gamma \dot{u}-A u=F(u, \dot{u}), \\
u(0)=u_{0}, \quad \dot{u}(0)=v_{0}
\end{array}\right.
$$

имеет единственное решение $u \in W_{\mathrm{bd}}^{2}\left(\mathbb{R}^{+}\right)$для любых $\left(u_{0}, v_{0}\right) \in \mathbb{K}^{+}$, и, следовательно, корректно определена разрешаюшая полугруппа

$$
\mathbb{S}_{h}: \mathbb{K}^{+} \rightarrow \mathbb{K}^{+}, \quad \mathbb{S}_{h}(u(0), \dot{u}(0))=(u(h), \dot{u}(h)),
$$

или, в эквивалентной форме, $\mathbb{S}_{h}=\Pi_{0} \mathscr{T}_{h}\left(\Pi_{0}\right)^{-1}$. Более того, сформулированное ниже следствие теоремы 2.5 показьвает, что полугруппа (2.16) непрерьвна по Гёльдеру в $\mathbb{K}^{+}$.

СлЕДСТВИЕ 2.6. Пусть выполнены условия теоремы 1.2 и условие (0.7). Тогда полугруппа (2.16) непрерывна по Гёльдеру с любой константой Гёльдера $\alpha$, принадлежащей интервалу $(0,1)$, т.е.

$$
\left\|\mathbb{S}_{h} z_{1}-\mathbb{S}_{h} z_{2}\right\|_{\mathbb{H}^{3 / 2}} \leqslant C_{\alpha} e^{M_{\alpha} h^{2}}\left\|z_{1}-z_{2}\right\|_{\mathbb{H}^{3 / 2}}^{1-\alpha}, \quad z_{1}, z_{2} \in \mathbb{K}^{+}
$$

где константыл $C_{\alpha}$ и $M_{\alpha}$ зависят от $\alpha u\left\|z_{i}\right\|_{\mathbb{H}^{3 / 2}}, i=1,2$.

ДокАЗАТЕльство. Действительно, выбрав в оценке (2.9) $t=h$ и $T=h / \alpha$ и использовав ограниченность решений $u_{1}$ и $u_{2}$ при $t \rightarrow \infty$, получим

$$
\left\|\mathbb{S}_{h} z_{1}-\mathbb{S}_{h} z_{2}\right\|_{\mathbb{H}^{1}} \leqslant C_{\alpha}^{\prime} e^{M_{\alpha}^{\prime} h^{2}}\left\|z_{1}-z_{2}\right\|_{\mathbb{H}^{1}}^{1-\alpha}, \quad z_{1}, z_{2} \in \mathbb{K}^{+} .
$$

Для вывода оценки (2.17) из (2.18) достаточно заметить, что из абстрактной теоремы о регулярности, примененной к уравнению (2.5), и теоремы о следах следует оценка

$$
\|v(h)\|_{H^{3 / 2}}+\|\dot{v}(h)\|_{H^{1 / 2}} \leqslant C\|v\|_{W^{2}(h)} \leqslant C_{1}\|v(0)\|_{H^{3 / 2}}+C_{2}\|v\|_{W^{1}(h, h+2)},
$$

в которой константы $C$ и $C_{1}$ зависят только от оператора $A$, а константа $C_{2}$ зависит также от константы $M$, определенной в (2.6). Объединяя оценки (2.18) и (2.19), получаем неравенство (2.17). Следствие 2.6 доказано.

Наша следуюшая задача - показать, что ДС $\left(\mathbb{S}_{h}\right)_{h \geqslant 0}$ в пространстве $\mathbb{K}^{+}$и траекторная ДС $\left(\mathscr{T}_{h}\right)_{h \geqslant 0}$ в пространстве $\mathscr{K}^{+}$являются топологически сопряженными при помоши гомеоморфизма $\Pi_{0}$. Очевидно, что для этого достаточно доказать непрерьвность $\left(\Pi_{0}\right)^{-1}$. Для проверки последнего факта мы введем следующую метрику в пространстве $\mathscr{K}^{+}$:

$$
d\left(u_{1}, u_{2}\right):=\sup _{T \in \mathbb{R}^{+}} e^{-T^{4}}\left\|u_{1}-u_{2}\right\|_{W^{2}(T)}
$$


С одной стороны, как нетрудно проверить, топология, индуцированная в $\mathscr{K}^{+}$метрикой $(2.20)$, совпадает с топологией, индуцированной вложением $\mathscr{K}^{+} \subset W_{\text {loc }}^{2}\left(\mathbb{R}^{+}\right)$ (благодаря ограниченности $\mathscr{K}^{+}{ }_{\text {в }} W_{\mathrm{bd}}^{2}\left(\mathbb{R}^{+}\right)$), а с другой стороны, из оценки (2.17) следует, что

$$
d\left(u_{1}, u_{2}\right) \leqslant C_{\alpha}^{\prime \prime}\left(\left\|u_{1}(0)-u_{2}(0)\right\|_{H^{3 / 2}}+\left\|\dot{u}_{1}(0)-\dot{u}_{2}(0)\right\|_{H^{1 / 2}}\right)^{1-\alpha}
$$

для любых $u_{1}, u_{2} \in \mathscr{K}^{+}$и любого $0<\alpha<1$. Здесь мы использовали также оценку

$$
\|v\|_{W^{2}(h)} \leqslant C\|v\|_{C\left(h, h+1 ; H^{3 / 2}\right) \cap C^{1}\left(h, h+1 ; H^{1 / 2}\right)}
$$

для решений уравнения (2.5), которая является элементарным следствием абстрактной теоремы о регулярности и оценки (2.6).

Таким образом, мы проверили, что полугруппы (2.16) и (2.1) действительно являются топологически сопряженными. Поэтому теорема 2.4 дает следующий результат.

ТеОРема 2.7. Пусть выполнены условия теоремы 1.2 и условие (0.7). Тогда полугруппа $\mathbb{S}_{h}$ обладает глобальным аттрактором $\mathbb{A}$ в $\mathbb{K}^{+}$, который описывается следующим образом:

$$
\mathbb{A}=\Pi_{0} \mathscr{A}^{\text {traj }}
$$

\section{§ 3. Колмогоровская энтропия аттрактора и ее оценки сверху}

В этом параграфе мы используем понятие колмогоровской $\varepsilon$-энтропии для изучения траекторного аттрактора уравнения (0.4), построенного в предыдушем параграфе. Мы начнем с напоминания определений $\varepsilon$-энтропии и фрактальной размерности. Подробное изложение этих вопросов можно найти в работе [21]. Приложения понятия колмогоровской энтропии к эволюционным уравнениям математической физики даны в работах [9], [10], [38], [41], [42].

ОПРЕДЕЛЕНИЕ 3.1. Пусть $M$ - метрическое пространство, а $K$ - предкомпактное множество в нем. Для любого $\varepsilon>0$ обозначим через $N_{\varepsilon}(K)=N_{\varepsilon}(K, M)$ наименьшее число $\varepsilon$-шаров в $M$, необходимое для покрытия множества $K$ (это число, очевидно, конечно, так как $K$ - предкомпакт). По определению, колмогоровской $\varepsilon$-энтропией множества $K$ в $M$ назьвается следуюшее число:

$$
\mathbf{H}_{\varepsilon}(K)=\mathbf{H}_{\varepsilon}(K, M) \equiv \ln N_{\varepsilon}(K) .
$$

ПримеР 3.2. Пусть $K$ - компактное $n$-мерное липшищево подмногообразие в $M$. Тогда очевидно, что $C_{1}(1 / \varepsilon)^{n} \leqslant N_{\varepsilon}(K) \leqslant C_{2}(1 / \varepsilon)^{n}$ и, следовательно, $\mathbf{H}_{\varepsilon}(K)=$ $\left(n+o(1)_{\varepsilon \rightarrow 0}\right) \ln (1 / \varepsilon)$.

Этот пример оправдьвает следуюшее определение.

ОПРЕДЕЛЕНИЕ 3.3. Фрактальной (әнтропийной) размерностью множества $K \Subset M$ назьвается следуюшее число:

$$
\operatorname{dim}_{\text {fract }}(K)=\operatorname{dim}_{\text {fract }}(K, M)=\limsup _{\varepsilon \rightarrow 0} \frac{\mathbf{H}_{\varepsilon}(K)}{\ln (1 / \varepsilon)} .
$$

Заметим, что построенный вьше аттрактор $\mathscr{A}$ уравнения $(0.4)$ компактен только в локальной топологии пространства $W_{\text {loc }}^{2}\left(\mathbb{R}^{+}\right)$, поэтому мы будем рассматривать $\varepsilon$-энтропию его ограничений $\left.\mathscr{A}\right|_{[0, T]}$ на конечный интервал $[0, T]$ и изучать ее зависимость от двух параметров $T$ и $\varepsilon$. Для этого нам понадобятся весовые аналоги пространств $W_{\mathrm{bd}}^{l}\left(\mathbb{R}^{+}\right)$. 
ОПРЕДЕЛЕНИЕ 3.4. По заданной весовой функции $\phi \in C(\mathbb{R},(0, \infty))$ определим пространство

$$
W_{\mathrm{bd}, \phi}^{l}\left(\mathbb{R}^{+}\right) \equiv\left\{u \in W_{\mathrm{loc}}^{l}\left(\mathbb{R}^{+}\right):\|u\|_{l, \mathrm{bd}, \phi} \equiv \sup _{T \in \mathbb{R}^{+}}\left\{\phi(T)\|u\|_{W^{l}(T)}\right\}<\infty\right\} .
$$

Следуя [31], [29], [13], [30], [42], мы введем также класс допустимых весовых функций.

ОПРЕДЕЛЕНИЕ 3.5. Функция $\phi \in L_{\mathrm{loc}}^{\infty}(\mathbb{R})$ назьвается весовой функцией с порядком роста $\mu \geqslant 0$, если сушествует $C>0$ такое, что

$$
\phi(x+y) \leqslant C e^{\mu|x|} \phi(y), \quad \phi(x)>0
$$

для любых $x, y \in \mathbb{R}$.

Как нетрудно проверить, из (3.4) следует также, что $\phi(x+y) \geqslant C^{-1} e^{-\mu|x|} \phi(y)$ для любых $x, y \in \mathbb{R}$.

Основньм результатом этого параграфа является следуюшая теорема.

ТЕОРема 3.6. Пусть выполнены условия теоремы 1.2. Тогда справедлива следующая оценка:

$$
\mathbf{H}_{\varepsilon}\left(\left.\mathscr{A}^{\text {traj }}\right|_{(0, T)}, W_{\mathrm{bd}}^{2}((0, T))\right) \leqslant C\left[T+\ln _{+} \frac{R_{0}}{\varepsilon}\right] \ln _{+} \frac{R_{0}}{\varepsilon},
$$

в которой $\ln _{+} \gamma:=\max \{0, \ln \gamma\}$, а константы $C$ и $R_{0}$ не зависят от $T u \varepsilon$.

Доказательство этой теоремы основано на серии промежуточных результатов и будет закончено после леммы 3.9 .

ПРЕДЛОЖЕНИЕ 3.7. Пусть $\mathscr{K} \subset W_{\mathrm{bd}}^{2}(\mathbb{R})$ - существенное множсество уравнения (0.4), определенное в теореме 2.4. Тогда для любого $\alpha>0$ существует константа $C=C(\alpha)$ такая, что для любых двух решений $u_{1}, u_{2} \in \mathscr{K}$ и любого $T \in \mathbb{R}$ справедлива оценка

$$
\left\|u_{1}-u_{2}\right\|_{W^{2}(T)} \leqslant C \sup _{t \in \mathbb{R}}\left\{e^{-\alpha|T-t|}\left\|u_{1}-u_{2}\right\|_{W^{0}(t)}\right\}
$$

ДокАЗАТЕльство. Действительно, определим функцию $v(t)=u_{2}(t)-u_{1}(t)$. Тогда $v$ удовлетворяет уравнению (2.5). Более того, из абстрактной внутренней оценки, примененной к этому уравнению, следует, что

$$
\|v\|_{W^{2}(T)} \leqslant C\left\|h_{v}\right\|_{W^{0}(T-1, T+2)}, \quad \text { где } \quad h_{v}:=L_{1}(t) v+L_{2}(t) \dot{v}
$$

$C$ не зависит от $T \in \mathbb{R}$. Умножив $(3.7)$ на $e^{-\alpha|T-M|}$ и взяв верхнюю грань по $T \in \mathbb{R}$ от обеих частей полученного неравенства, мы выведем следующий аналог теоремы о регулярности в весовых пространствах $W_{\mathrm{bd}, e^{-\alpha|T-M|}}^{2}$ :

$$
\sup _{T \in \mathbb{R}}\left\{e^{-\alpha|T-M|}\|v\|_{W^{2}(T)}\right\} \leqslant C_{1} \sup _{T \in \mathbb{R}}\left\{e^{-\alpha|T-M|}\left\|h_{v}\right\|_{W^{0}(T)}\right\}
$$


где константа $C_{1}$ не зависит $M$. Таким образом, остается оценить правую часть неравенства (3.8). Для этого мы заметим, что из условия $(0.5)(\mathrm{a})$ и ограниченности $\mathscr{K}$ в $W_{\mathrm{bd}}^{2}(\mathbb{R})$ следует оценка

$$
\left\|L_{1}(t)\right\|_{H^{3 / 2} \rightarrow H}+\left\|L_{2}(t)\right\|_{H^{1 / 2} \rightarrow H} \leqslant R_{*},
$$

где $R_{*}$ не зависит от $t$ и $u_{1}, u_{2} \in \mathscr{K}$. Следовательно, согласно (3.9) и интерполяционным неравенствам (см. [35]),

$$
\left\|h_{v}(t)\right\|_{W^{0}(T)} \leqslant C_{2} R_{*}\|v\|_{W^{3 / 2}(T)} \leqslant \mu\|v\|_{W^{2}(T)}+C_{\mu}\|v\|_{W^{0}(T)},
$$

где $\mu>0$ произвольно, а $C_{\mu}>0$ - положительная константа, зависящая от $\mu$, но не зависящая от $T$ и $u_{1}, u_{2} \in \mathscr{K}$. Зафиксировав $\mu=1 /\left(2 C_{1}\right)$ в неравенстве $(3.10)$ и подставив его в (3.8), получим

$$
\sup _{T \in \mathbb{R}}\left\{e^{-\alpha|T-M|}\|v\|_{W^{2}(T)}\right\} \leqslant 2 C_{1} C_{\mu} \sup _{T \in \mathbb{R}}\left\{e^{-\alpha|T-M|}\|v\|_{W^{0}(T)}\right\} .
$$

Очевидное неравенство

$$
\|v\|_{W^{2}(M)} \leqslant e^{-\alpha} \sup _{T \in \mathbb{R}}\left\{e^{-\alpha|T-M|}\|v\|_{W^{2}(T)}\right\}
$$

завершает доказательство предложения 3.7.

Рассмотрим теперь семейство весовых функций

$$
\phi_{R}(t)= \begin{cases}1 & \text { при }|t|<R, \\ e^{R-|t|} & \text { при }|t| \geqslant R,\end{cases}
$$

где $R \in \mathbb{R}^{+}$. Эти функции, очевидно, удовлетворяют неравенству (3.4) с константами $\mu=1$ и $C_{\phi}=1$, которые не зависят, таким образом, от $R$.

С ЛЕДСТВИЕ 3.8. Пусть $\mathscr{K} \subset W_{\mathrm{bd}}^{2}(\mathbb{R})$ - существенное множество уравнения (0.4). Тогда существует константа $C_{\mathscr{K}}>0$ такая, что для любых $u_{1}, u_{2} \in K$ и любого $R>0$ справедлива следующая оценка:

$$
\left\|u_{1}-u_{2}\right\|_{W_{\mathrm{bd}, \phi_{R}}^{2}(\mathbb{R})} \leqslant C_{\mathscr{K}}\left\|u_{1}-u_{2}\right\|_{L_{\mathrm{bd}, \phi_{R}}^{2}(\mathbb{R})} .
$$

Действительно, фиксировав $\alpha=2$ в неравенстве (3.6), умножив его на $\phi_{R}(T)$ и применив $\sup _{T \in \mathbb{R}}$ K обеим частям полученного неравенства, после несложных преобразований мы получим оценку (3.14) (см. [42]). Более того, так как $\phi_{R}$ удовлетворяют (3.4) равномерно по $R>0$, то константа $C_{\mathscr{K}}$ в $(3.14)$ также не зависит от $R$.

Заметим, что весовые функции $\phi_{R}$ выбраны таким образом, чтобы

$$
\left\|\left.v\right|_{(0, R)}\right\|_{W_{\mathrm{bd}}^{2}((0, R))} \leqslant\|v\|_{W_{\mathrm{bd}, \phi_{R}}^{2}(\mathbb{R})}
$$

для любых $v \in W_{\mathrm{bd}}^{2}(\mathbb{R})$, следовательно,

$$
\mathbf{H}_{\varepsilon}\left(\left.\mathscr{A}^{\mathrm{traj}}\right|_{(0, R)}, W_{\mathrm{bd}}^{2}((0, R))\right) \leqslant \mathbf{H}_{\varepsilon}\left(K, W_{\mathrm{bd}, \phi_{R}}^{2}(\mathbb{R})\right) .
$$

Таким образом, для оценки энтропии множеств $\left.\mathscr{A}\right|_{(0, R)}$, следуя [42], мы будем оценивать сверху энтропию существенного множества $\mathscr{K}$ уравнения (0.4) в весовых пространствах $W_{\mathrm{bd}, \phi_{R}}^{2}(\mathbb{R})$. Для этого нам понадобится следующая лемма, доказательство которой основано на оценке $(3.14)$ и компактности вложения $W_{\mathrm{bd}}^{2}(\mathbb{R})$ в $L_{\mathrm{bd}, \phi_{R}}^{2}(\mathbb{R})$. 
Лемма 3.9. Энтропия существенного множества Ж уравнения (0.4) удовлетворяет следующей рекуррентной оценке:

$$
\mathbf{H}_{\varepsilon / 2}\left(\mathscr{K}, W_{\mathrm{bd}, \phi_{R}}^{2}(\mathbb{R})\right) \leqslant L\left[R+1+\ln _{+} \frac{R_{0}^{\prime}}{\varepsilon}\right]+\mathbf{H}_{\varepsilon}\left(\mathscr{K}, W_{\mathrm{bd}, \phi_{R}}^{2}(\mathbb{R})\right),
$$

в которой константы $L$ и $R_{0}^{\prime}$ не зависят от $R>0 u \varepsilon>0$.

ДоказАТельство. Пусть $\left\{B\left(u^{i}, \varepsilon, W_{\mathrm{bd}, \phi_{R}}^{2}\right): i=1, \ldots, N_{\varepsilon}\right\}-\varepsilon$-покрытие множества $\mathscr{K}$ (здесь и далее через $B(v, \mu, X)$ обозначается шар радиуса $\mu$ с центром в точке $v$ в метрике пространства $X)$. Заметим, что $\mathscr{K} \cap B\left(u^{i}, C \varepsilon, W_{\mathrm{bd}, \phi_{R}}^{2}(\mathbb{R})\right)$ компактно в $L_{\mathrm{bd}, \phi_{R}}^{2}(\mathbb{R})$, следовательно, каждое такое множество может быть покрыто конечньм числом $\varepsilon /\left(2 C_{\mathscr{K}}\right)$-шаров $\left\{B\left(u^{i, j}, \varepsilon /\left(2 C_{\mathscr{K}}\right), L_{\mathrm{bd}, \phi_{R}}^{2}(\mathbb{R})\right), j=1, \ldots, \mathscr{M}_{i}(\varepsilon)\right\}$, где $C_{\mathscr{K}}$ такое же, как и в $(3.14)$, а

$$
\mathscr{M}_{i}(\varepsilon):=N_{\varepsilon /\left(2 C_{\mathscr{K}}\right)}\left(\mathscr{K} \cap B\left(u^{i}, \varepsilon, W_{\mathrm{bd}, \phi_{R}}^{2}(\mathbb{R})\right), L_{\mathrm{bd}, \phi_{R}}^{2}(\mathbb{R})\right) .
$$

Из оценки (3.14) следует теперь, что система шаров $\left\{B\left(u^{i, j}, \varepsilon / 2, W_{\mathrm{bd}, \phi_{R}}^{2}(\mathbb{R})\right): i=\right.$ $\left.1, \ldots, N_{\varepsilon}, j=1, \ldots, \mathscr{M}_{i}(\varepsilon)\right\}$ является $\varepsilon / 2$-покрытием $\mathscr{K}$, что приводит к рекуррентной оценке

$$
\mathbf{H}_{\varepsilon / 2}\left(\mathscr{K}, W_{\mathrm{bd}, \phi_{R}}^{2}(\mathbb{R})\right) \leqslant \max _{i=1, \ldots, N_{\varepsilon}} \ln \mathscr{M}_{i}(\varepsilon)+\mathbf{H}_{\varepsilon}\left(\mathscr{K}, W_{\mathrm{bd}, \phi_{R}}^{2}(\mathbb{R})\right) .
$$

Таким образом, остается оценить величины $\mathscr{M}_{i}(\varepsilon)$, введенные в (3.18). Для этого заметим, что, согласно $(1.3),\|u\|_{W_{\mathrm{bd}}^{2}(\mathbb{R})} \leqslant C_{*}$ для любого $u \in \mathscr{K}$, следовательно,

$$
\|u\|_{W_{\phi_{R}}^{2}(T)} \leqslant \frac{\varepsilon}{4 C_{\mathscr{K}}} \text { при }|T| \geqslant T_{\varepsilon} \equiv R+\ln _{+} \frac{4 C_{*} C_{\mathscr{K}}}{\varepsilon} .
$$

Поэтому

$$
\begin{aligned}
\mathscr{M}_{i}(\varepsilon) & \leqslant N_{\varepsilon /\left(4 C_{\mathscr{K}}\right)}\left(\mathscr{K} \cap B\left(u^{i}, \varepsilon, W_{\mathrm{bd}, \phi_{R}}^{2}(\mathbb{R})\right), L_{\mathrm{bd}, \phi_{R}}^{2}\left(\left(-T_{\varepsilon}, T_{\varepsilon}\right)\right)\right) \\
& \leqslant N_{\varepsilon /\left(4 C_{\mathscr{K}}\right)}\left(B\left(u^{i}, \varepsilon, W_{\mathrm{bd}, \phi_{R}}^{2}\left(\left(-T_{\varepsilon}, T_{\varepsilon}\right)\right)\right), L_{\mathrm{bd}, \phi_{R}}^{2}\left(\left(-T_{\varepsilon}, T_{\varepsilon}\right)\right)\right) \\
& \leqslant N_{1 /\left(4 C_{\mathscr{K}}\right)}\left(B\left(0,1, W_{\mathrm{bd}, \phi_{R}}^{2}\left(\left(-T_{\varepsilon}, T_{\varepsilon}\right)\right)\right), L_{\mathrm{bd}, \phi_{R}}^{2}\left(\left(-T_{\varepsilon}, T_{\varepsilon}\right)\right)\right) .
\end{aligned}
$$

В первой оценке была использована оценка "хвостов" (3.20), во второй оценке мы убрали символ “ $\mathscr{K} \cap$ ”, увеличив при этом значение $N_{\varepsilon /\left(4 C_{\mathscr{K}}\right)}(\ldots)$, а в третьей оценке мы воспользовались гомотетией и трансляионной инвариантностью шаров в банаховых пространствах.

Итак, остается оценить энтропию оператора вложения

$$
W_{\mathrm{bd}, \phi_{R}}^{2}((-T, T)) \subset L_{\mathrm{bd}, \phi_{R}}^{2}((-T, T)) .
$$

Для этой цели мы введем гладкий аналог $\psi_{R} \in C^{\infty}(\mathbb{R})$ весовых функций $\phi_{R}$ такой, что

$$
\max \left\{\left|\dot{\psi}_{R}(t)\right|,\left|\ddot{\psi}_{R}(t)\right|\right\} \leqslant \psi_{R}(t) \text { и } C^{\prime} \phi_{R}(t) \leqslant \psi_{R}(t) \leqslant C^{\prime \prime} \phi_{R}(t)
$$


где константы $C^{\prime}$ и $C^{\prime \prime}$ не зависят от $R$. Тогда, как нетрудно проверить, отображение $\mathbb{F}_{R}: u \rightarrow \psi_{R}^{1 / 2} u$ реализует линейный изоморфизм банаховых пар

$$
\begin{aligned}
\mathbb{B}(T) & =\left(W_{\mathrm{bd}}^{2}((-T, T)), L_{\mathrm{bd}}^{2}((-T, T))\right), \\
\mathbb{B}_{R}(T) & =\left(W_{\mathrm{bd}, \phi_{R}}^{2}((-T, T)), L_{\mathrm{bd}, \phi_{R}}^{2}((-T, T))\right) .
\end{aligned}
$$

Более того,

$$
\left\|\mathbb{F}_{R}\right\|_{\mathbb{B}(T) \rightarrow \mathbb{B}_{R}(T)}+\left\|\mathbb{F}_{R}^{-1}\right\|_{\mathbb{B}_{R}(T) \rightarrow \mathbb{B}(T)} \leqslant C_{2},
$$

где $C_{2}$ не зависит от $T$ и $R$ (см. [42]). Таким образом,

$$
\begin{aligned}
\ln \mathscr{M}_{i}(\varepsilon) & \leqslant \mathbf{H}_{1 /\left(4 C_{\mathscr{K}}\right)}\left(B\left(0,1, W_{\mathrm{bd}, \phi_{R}}^{2}\left(\left(-T_{\varepsilon}, T_{\varepsilon}\right)\right)\right), L_{\mathrm{bd}, \phi_{R}}^{2}\left(\left(-T_{\varepsilon}, T_{\varepsilon}\right)\right)\right) \\
& \leqslant \mathbf{H}_{1 /\left(4 C_{\mathscr{K}} C_{2}^{2}\right)}\left(B\left(0,1, W_{\mathrm{bd}}^{2}\left(\left(-T_{\varepsilon}, T_{\varepsilon}\right)\right)\right), L_{\mathrm{bd}}^{2}\left(\left(-T_{\varepsilon}, T_{\varepsilon}\right)\right)\right) .
\end{aligned}
$$

Очевидная оценка

$$
\begin{aligned}
& \mathbf{H}_{\mu}\left(B\left(0,1, W_{\mathrm{bd}}^{2}((-T, T))\right), L_{\mathrm{bd}}^{2}((-T, T))\right) \\
& \quad \leqslant(2 T+1) \mathbf{H}_{\mu / 2}\left(B\left(0,1, W_{\mathrm{bd}}^{2}((-1,1))\right), L_{\mathrm{bd}}^{2}((-1,1))\right)
\end{aligned}
$$

заканчивает теперь доказательство леммы 3.9 .

ЗАВЕРШЕНИЕ ДОКАЗАТЕЛЬСТВА ТЕОРЕМЫ 3.6. Согласно (1.3),

$$
\mathbf{H}_{C_{*}}\left(\mathscr{K}, W_{\mathrm{bd}, \phi_{R}}^{2}(\mathbb{R})\right)=0 \text { для любого } R \geqslant 0 .
$$

Итерируя оценку (3.17) $k$ раз, получим

$$
\mathbf{H}_{2^{-k} C_{*}}\left(\mathscr{K}, W_{\mathrm{bd}, \phi_{R}}^{2}(\mathbb{R})\right) \leqslant L\left(R+1+\ln _{+} \frac{R_{0}^{\prime} 2^{k-1}}{C_{*}}\right) k \text { для любого } k \in \mathbb{N} \text {. }
$$

По заданному $\varepsilon>0$ мы выберем $k$ такое, что $2^{-k} C_{*} \leqslant \varepsilon<2^{-k+1} C_{*}$. Подставив это значение $k$ в оценку (4.25), мы получим оценку (3.5). Теорема 3.6 доказана.

Мы завершим этот параграф̆ приложением теоремы 3.6 к изучению эллиптической краевой задачи $(0.6)$ в цилиндрической области $\Omega_{+}=\mathbb{R}^{+} \times \omega$, где $\omega \Subset \mathbb{R}^{n}-$ гладкая ограниченная область. Для простоты мы сформулируем условия на нелинейную функцию $f(u, \dot{u})$ только для случая $n \leqslant 3$. Эти условия имеют следуюший вид:

$$
\left\{\begin{array}{l}
\text { (1) } f \in C^{1}\left(\mathbb{R}^{k} \times \mathbb{R}^{k}, \mathbb{R}^{k}\right) \\
\text { (2) } f(u, v) \cdot u \geqslant-C, \mathrm{D}_{u} f(u, v) \geqslant-C \\
\text { (3) }|f(u, v)|+\left|\mathrm{D}_{u} f(u, v)\right| \leqslant C\left(1+|u|^{k_{1}}\right)\left(1+|v|^{k_{2}}\right), \\
\text { (4) }\left|\mathrm{D}_{v} f(u, v)\right| \leqslant C\left(1+|u|^{k_{1}}\right),
\end{array}\right.
$$

где $0 \leqslant k_{2}<1$ и $0 \leqslant k_{1}<\frac{n+3}{n-1}\left(1-k_{2}\right)$. Заметим, что в случае, когда нелинейность не зависит явно от $\partial_{t} u$ (и, следовательно, $\left.k_{2}=0\right)$, мы получаем стандартные ограничения на рост нелинейности $f(u)$, сформулированные в работе [2]. 
СлЕДСТВИЕ 3.10. Пусть $\gamma=\gamma^{*} \in \mathscr{L}\left(\mathbb{R}^{k}, \mathbb{R}^{k}\right), g \in L^{2}(\omega), n \leqslant 3$, а нелинейность $f$ удовлетворяет условиям (3.26). Тогда уравнение (0.6) удовлетворяет всем условиям теоремы 3.6 и, следовательно, обладает траекторным аттрактором $\mathscr{A}^{\text {traj }}$ энтропия которого допускает оченки сверху вида (3.5).

ДокАЗАТЕльство. Перепишем уравнение (0.6) в абстрактной форме (0.4). Действительно, в этом случае $H=L^{2}(\omega), A=-\Delta_{x}$ (с граничными условиями Дирихле) и $F(u, v):=f(u, v)+g$. Следовательно, необходимо проверить, что определенный так оператор $F(u, v)$ удовлетворяет условиям (0.5). Для этого мы напомним, что пространства $H^{s}:=D\left(\left(-\Delta_{x}\right)^{-s / 2}\right)$ удовлетворяют вложению $H^{s} \subset H^{s}(\omega)$ при $s \geqslant 0$, где $H^{s}(\omega)$ - классические соболевские пространства (см., например, [35]). Заметим также, что в случае $n \leqslant 3$ справедливо вложение $H^{3 / 2} \subset L^{p}$ для любого $p<\infty$, а следовательно, для любого фиксированного $p<\infty$ сушествует $\delta=\delta(p)>0$ такое, что $H^{3 / 2-\delta} \subset L^{p}$.

Проверим условие (0.5)(a). Действительно, согласно (3.26)(3) и неравенству Гёльдера,

$$
\begin{aligned}
\left\|\mathrm{D}_{u} F(u, v) \theta\right\|_{L^{2}}^{2} & \leqslant C\left\|\left(1+|u|^{2 k_{1}}\right)\left(1+|v|^{2 k_{2}}\right)|\theta|^{2}\right\|_{L^{1}} \\
& \leqslant\left(1+\|v\|_{L^{2}}^{2 k_{2}}\right)\left(1+\|u\|_{L^{4 k_{1} /\left(1-k_{2}\right)}}^{2 k_{1}}\right)\|\theta\|_{L^{4 /\left(1-k_{2}\right)}}^{2} \\
& \leqslant Q\left(\|u\|_{H^{3 / 2-\delta}}+\|v\|_{L^{2}}\right)\|\theta\|_{H^{3 / 2}-\delta}^{2}
\end{aligned}
$$

при достаточно малом $\delta>0$. Оценка

$$
\left\|\mathrm{D}_{v} F(u, v) \theta\right\| \leqslant Q\left(\|u\|_{H^{3 / 2-\delta}}+\|v\|_{H^{1 / 2-\delta}}\right)\|\theta\|_{H^{1 / 2-\delta}}
$$

доказывается аналогично. Непрерывность операторов $F, \mathrm{D}_{u} F$ и $\mathrm{D}_{v} F$ вьводится стандартным образом из условия (3.26)(1). Таким образом, условие (0.5)(a) проверено. Условия $(0.5)(\mathrm{b}),(0.5)(\mathrm{c})$ являются немедленньми следствиями предположения $(3.26)(2)$. Поэтому остается вывести условие $(0.5)(\mathrm{d})$ из $(3.26)(3)$. Действительно, согласно неравенству Гёльдера и теореме вложения Соболева,

$$
\|F(u, v)\|_{L^{2}}^{2} \leqslant C\left(1+\|v\|_{L^{2}}^{2}\right)+\|u\|_{L^{p}}^{p} \leqslant C\left(1+\|v\|_{L^{2}}^{2}\right)+C_{1}\|u\|_{H^{s}(\omega)}^{p},
$$

где $p:=2 k_{1} /\left(1-k_{2}\right)$ и $1 / p=1 / 2-s / n$. Если $s \leqslant 1 / 2$, то $(0.5)(\mathrm{d})$ немедленно следует из (3.27). Предположим, что $s \geqslant 1 / 2$. Тогда, согласно интерполящионному неравенству,

$$
\|u\|_{H^{s}(\omega)} \leqslant C_{2}\|u\|_{H^{2}(\omega)}^{(2 s-1) / 3}\|u\|_{H^{1 / 2}(\omega)}^{(4-2 s) / 3} .
$$

Заметим, что $(0.5)(\mathrm{d})$ следует из $(3.27)$ и $(3.28)$, если $p(2 s-1) / 3<2$. Поэтому достаточно проверить это неравенство. Заметим, что, согласно нашим предположениям, $p<2(n+3) /(n-1)$, следовательно, $s<2 n /(n+3)$, а значит, $p(2 s-1) / 3<$ $2(n+3) / 3(n-1) \cdot 3(n-1) /(n+3)=2$. Итак, условие $(0.5)(\mathrm{d})$ также проверено. Следствие 3.10 доказано. 


\section{§ 4. Энтропия аттрактора: пример точной оценки снизу}

В этом параграфе мы построим пример уравнения (0.4), для которого оценка энтропии (3.5) является, в некотором смысле, точной. Более того, фрактальная размерность аттрактора $\mathbb{A}$ оказьвается бесконечной. Этот пример существенно использует контрпример к теории Флоке для абстрактных эллиптических краевых задач в цилиндрических областях, построенный в работах [27], [12].

Рассмотрим следующее линейное эллиптическое уравнение в полосе $\mathbb{R} \times \omega \equiv$ $\mathbb{R} \times(0, \pi):$

$$
\ddot{u}+\partial_{x}^{2} u=L_{1}(t) u+L_{2}(t) \dot{u},\left.\quad u\right|_{\mathbb{R} \times \partial \omega}=0 .
$$

Здесь $L_{1}(t)$ и $L_{2}(t)$ - линейные операторы, которые являются $T$-периодическими по $t$. Основная идея вышеупомянутого контрпримера - построить операторы $L_{1}$ и $L_{2}$ таким образом, чтобы уравнение (4.1) имело решение $u$, которое убьвало бы быстрее любой экспоненты при $t \rightarrow \pm \infty$. Этот факт, очевидно, противоречит теории Флоке, при наличии которой решение должно быть линейной комбинацией произведений экспонент и периодических функций. Точнее, следуюший результат доказан в монографии [12] (см. приложение А, с. 261-262).

Теорема 4.1. Существуют T-периодические операторы $L_{j}(t)$, удовлетворяющие условиям

$L_{1} \in C_{\mathrm{bd}}^{\infty}\left(\mathbb{R}, \mathscr{L}\left(H^{s+1}, H^{s}\right)\right)$ u $L_{2} \in C_{\mathrm{bd}}^{\infty}\left(\mathbb{R}, \mathscr{L}\left(H^{s}, H^{s}\right)\right)$ для любого $s \in \mathbb{R}$

и $L_{j}(t+T)=L_{j}(t)$ для любых $t \in \mathbb{R}$, такие, что уравнение (4.1) обладает решением $u \in W^{2}(\mathbb{R})$, которое допускает следующую оценку:

$$
C_{1} e^{-\beta t^{2}} \leqslant\|u(t)\|_{L^{2}(\omega)} \leqslant C_{2} e^{-t^{2}} \quad \text { npu } \quad t \in \mathbb{R}
$$

для некоторых положительных констант $\beta, C_{1} u C_{2}$. Более того, все коэффициенты Фурье $u_{n}(t)=\int_{0}^{\pi} u(t, x) \sin (n x) d x$ этого решения имеют компактнье носители в $\mathbb{R}$. ( Мы обозначаем здесь символом $H^{s}$ шкалу пространств, порожсденную лапласианом в $\omega$ с граничнылми условиями Дирихле.)

Заметим, что не только функщия $u$, но и все функции, полученные из $u$ сдвигами на $k T, k \in \mathbb{Z}$ (т.е. $\mathscr{T}_{k T} u: t \mapsto u(t+k T)$ ), являются линейно независимыми решениями уравнения (4.1), и, следовательно, линейньй эллиптический оператор в $L^{2}(\mathbb{R} \times \omega)$, определенньй уравнением (4.1), имеет бесконечномерное ядро. Определим множество

$$
\mathbb{L} \equiv\left\{v=\sum_{k \in \mathbb{Z}} a_{k} \mathscr{T}_{k T} u: a_{k} \in \mathbb{R}, \sup _{k \in \mathbb{Z}}\left|a_{k}\right|<\infty\right\} .
$$

Тогда справедливо следующее утверждение.

Лемма 4.2. Пусть и -решение, построенное в теореме 4.1, а $\mathbb{L}$ определено формулой (4.4). Предположим также, что носитель первого коэффичиента Фурье $u_{1}: t \mapsto \int_{0}^{\pi} u(t, x) \sin x d x$ функиии и удовлетворяет условию $\varnothing \neq \operatorname{supp} u_{1} \subset$ $[r, r+N]$ для некоторого $r \in \mathbb{R} u N \in(0, T)$. Тогда

$$
C_{1} \sup _{k \in \mathbb{Z}}\left|a_{k}\right| \leqslant\|v\|_{W_{\text {bd }}^{2}(\mathbb{R})} \leqslant C_{2} \sup _{k \in \mathbb{Z}}\left|a_{k}\right|
$$

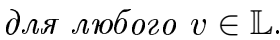


ДокАЗАТЕльство. Действительно, левое неравенство в (4.5) следует из того, что множества supp $\mathscr{T}_{k T} u_{1}$ не пересекаются при разных $k$.

Для доказательства правого неравенства заметим, что из (4.3) и регулярности решений эллиптических уравнений следует оценка

$$
\|u\|_{W^{2}(\tau)} \leqslant C_{2} e^{-\tau^{2} / 2}
$$

для решения $u$. Таким образом,

$$
\|v\|_{W^{2}(\tau)} \leqslant \sum_{k \in \mathbb{Z}}\left|a_{k}\right|\|u\|_{W^{2}(\tau-k T)} \leqslant \sum_{k \in \mathbb{Z}}\left|a_{k}\right| C_{2} e^{-(\tau-k T)^{2} / 2} \leqslant C \sup _{k \in \mathbb{Z}}\left|a_{k}\right| .
$$

Лемма 4.2 доказана.

Заменив период $T$ на $l T, l \in \mathbb{N}$, если это необходимо, мы можем считать, что условие леммы 4.2 на носитель supp $u_{1}$ выполнено для функции $u$, введенной в теореме 4.1.

ЛЕМмА 4.3. Пусть выполнены условия леммы 4.2, и пусть

$$
\mathbb{L}_{R}=\left\{v \in \mathbb{L}:\|v\|_{W_{\mathrm{bd}}^{2}(\mathbb{R})} \leqslant R\right\}
$$

Тогда существуют положительные константы $C(R), M_{0}$ и $\varepsilon_{0}$ такие, что при $M \geqslant M_{0} u \varepsilon \in\left(0, \varepsilon_{0}\right)$ справедлива оченка снизу

$$
\mathbf{H}_{\varepsilon}\left(\left.\mathbb{L}_{R}\right|_{(0, M)}, W_{\mathrm{bd}}^{2}((0, M))\right) \geqslant C(R) M \ln \frac{1}{\varepsilon} .
$$

ДокаЗАТЕльство. Без ограничения общности можно считать, что supp $u_{1} \subset$ $[0, T]$. Пусть $\mathbb{L}_{R}^{k}=\left\{v \in \mathbb{L}_{R}: v(t)=\sum_{i=1}^{k} a_{k} u(t-i T)\right\}$, тогда для любых $v^{1}, v^{2} \in \mathbb{L}_{R}^{k}$ справедлива оценка

$$
\left\|v^{1}-v^{2}\right\|_{W_{b}^{2}((0,(k+1) T))} \geqslant\left\|v^{1}-v^{2}\right\|_{C([0,(k+1) T], H)} \geqslant K \sup _{i=1, \ldots, k}\left|a_{i}^{1}-a_{i}^{2}\right|,
$$

где $K=\left\|u_{1}\right\|_{C([0, T])}>0$.

Для любого достаточно большого $M$ выберем $k=k_{M}$ как решение неравенства $(k+1) T>M \geqslant k T$. Тогда правая оценка (4.5) гарантирует, что функция $v \in \mathbb{L}$ принадлежит $\mathbb{L}_{R}$, если все коэффициенты $\left|a_{k}\right| \leqslant R / C_{2}$. Более того, согласно (4.10), две функщии $v_{1}$ и $v_{2}$ из $\mathbb{L}_{R}^{k} \varepsilon$-различимы, если $\left|a_{i}^{1}-a_{i}^{2}\right| \geqslant \varepsilon / K$ хотя бы для одного $i \in\{0, \ldots, k\}$. Следовательно,

$$
N_{\varepsilon / 2}\left(\mathbb{L}_{R}^{k}, W_{\mathrm{bd}}^{2}([0, M])\right) \geqslant\left(2\left[\frac{K R}{C_{2} \varepsilon}\right]+1\right)^{k} .
$$

Так как $k \sim M$, то (4.11) доказьвает лемму 4.3.

Теперь мы готовы построить уравнение вида (0.4), удовлетворяющее условиям теоремы 3.6, таким образом, чтобы его аттрактор $\mathscr{A}$ содержал множество $\Pi_{+} \mathbb{L}_{R}$ при достаточно малом $R$. Тогда лемма 4.3 даст необходимую оценку снизу $\varepsilon$-энтропии аттрактора. 
Пусть операторы $L_{1}$ и $L_{2}$ - такие же, как и в теореме 4.1. Так как эти операторы $T$-периодичны (для простоты мы считаем далее, что $T=2 \pi$ ), то существуют гладкие семейства операторов $\widehat{L}_{1} \in C^{\infty}\left(\mathbb{R}^{2}, \mathscr{L}\left(H^{s+1}, H^{s}\right)\right)$ и $\widehat{L}_{2} \in C^{\infty}\left(\mathbb{R}^{2}, \mathscr{L}\left(H^{s}, H^{s}\right)\right)$ такие, что

$$
\widehat{L}_{1}\left(w_{1}, w_{2}\right)=\widehat{L}_{2}\left(w_{1}, w_{2}\right)=0 \text { при }\left|w_{1}\right|^{2}+\left|w_{2}\right|^{2} \geqslant 2
$$

$$
L_{1}(t)=\widehat{L}_{1}(\cos t, \sin t), \quad L_{2}(t)=\widehat{L}_{2}(\cos t, \sin t) .
$$

Более того, пара $w(t)=(\cos t, \sin t)$ может быть получена как решение системы ОДУ

$$
\ddot{w}-w=\frac{2\left(|w|^{2} w-3 w\right)}{1+|w|^{4}} .
$$

Пусть $\phi_{R}(z): \mathbb{R} \rightarrow \mathbb{R}$ - срезающая функция такая, что $\phi_{R}=1$ при $|z| \leqslant R^{2}$ и $\phi_{R}=0$ при $|z| \geqslant 2 R^{2}$. Рассмотрим следуюшую систему:

$$
\left\{\begin{array}{l}
\ddot{w}-w=2\left(|w|^{2} w-3 w\right) /\left(1+|w|^{4}\right), \\
\ddot{u}+\partial_{x}^{2} u=\phi_{R}\left(\|u\|_{H^{1}(\omega)}^{2}+\|\dot{u}\|_{L^{2}(\omega)}^{2}\right)\left[\widehat{L}_{1}\left(w_{1}, w_{2}\right) u+\widehat{L}_{2}\left(w_{1}, w_{2}\right) \dot{u}\right] .
\end{array}\right.
$$

Тогда, с одной стороны, из вложения $W_{\mathrm{bd}}^{2}(\mathbb{R}) \subset C_{\mathrm{bd}}\left(\mathbb{R}, H^{1}\right) \cap C_{\mathrm{bd}}^{1}(\mathbb{R}, H)$ следует неравенство

$$
\|u(t)\|_{H^{1}}^{2}+\|\dot{u}(t)\|_{L^{2}}^{2} \leqslant P^{2}\|u\|_{W_{\mathrm{bd}}^{2}(\mathbb{R})}^{2}
$$

для некоторой положительной константы $P$, и, следовательно, существенное множество $\mathscr{K}$ этой системы содержит $\{(\cos t, \sin t)\} \times \mathbb{L}_{R / P}$ в качестве подмножества. С другой стороны, это уравнение удовлетворяет условиям $(0.5)$ и $(0.7)$ при $H=\mathbb{R}^{2} \times L^{2}(\omega)$, $A=\operatorname{diag}\left\{1,1,-\partial_{x}^{2}\right\}, \gamma=0$ и

$$
F=\left(\begin{array}{c}
2\left(|w|^{2} w-3 w\right) /\left(1+|w|^{4}\right) \\
\phi_{R}\left(\|u\|_{H^{1}(\omega)}^{2}+\|\dot{u}\|_{L^{2}(\omega)}^{2}\right)\left[\widehat{L}_{1}\left(w_{1}, w_{2}\right) u+\widehat{L}_{2}\left(w_{1}, w_{2}\right) \dot{u}\right]
\end{array}\right) .
$$

Действительно, из определения операторов $\widehat{L}_{i}, i=1,2$, следует, что

$$
\left\|\widehat{L}_{1}\left(w_{1}, w_{2}\right) u\right\|_{L^{2}}+\left\|\widehat{L}_{2}\left(w_{1}, w_{2}\right) \dot{u}\right\|_{L^{2}} \leqslant C\left(\|u\|_{H^{1}}+\|\dot{u}\|_{L^{2}}\right),
$$

где константа $C$ не зависит от $w$, и, следовательно, $\|F(w, u, \dot{u})\|_{L^{2}} \leqslant C_{R}^{\prime}$, где $C_{R}^{\prime}$ не зависит от $w, u$ и $\dot{u}$. Таким образом, условия $(0.5)(\mathrm{c})$ и $(0.5)(\mathrm{d})$ проверены. Аналогично, из гладкости $\widehat{L}_{i}$ по $w$ и выбора срезающей функции $\phi_{R}$ следует, что

$$
\left\|\mathrm{D}_{w} F(w, u, \dot{u})\right\|_{\mathbb{R}^{2} \rightarrow H}+\left\|\mathrm{D}_{u} F(w, u, \dot{u})\right\|_{H^{1} \rightarrow H}+\left\|\mathrm{D}_{\dot{u}} F(w, u, \dot{u})\right\|_{H^{0} \rightarrow H} \leqslant C_{R}^{\prime \prime},
$$

где $C_{R}^{\prime \prime}$ также не зависит от $w, u$ и $\dot{u}$. Поэтому условия $(0.5)(\mathrm{a})$ и $(0.7)$ также вьполняются. Остается заметить, что условие $(0.5)(\mathrm{b})$ также является немедленным следствием последней оценки.

Итак, мы доказали, что нелинейность $F(w, u, \dot{u})$ действительно удовлетворяет условиям (0.5) и (0.7) и, следовательно, уравнение (4.15) удовлетворяет условиям теорем 2.7 и 3.6. В частности, объединив утверждения теоремы 3.6 и леммы 4.3 , получим следующую двустороннюю оценку для $\varepsilon$-энтропии его аттрактора. 
ТЕОРема 4.4. Уравнение (4.15) обладает траекторным аттрактором $\mathscr{A}^{\text {traj, }}$ и существуют положительные константы $T_{0}, \varepsilon_{0}, C_{1}$ и $C_{2}$ такие, что в-энтропия $\mathscr{A}^{\text {traj }}$ удовлетворяет оценкам

$$
C_{1} T \ln \frac{1}{\varepsilon} \leqslant \mathbf{H}_{\varepsilon}\left(\left.\mathscr{A}^{\operatorname{traj}}\right|_{(0, T)}, W_{\mathrm{bd}}^{2}((0, T))\right) \leqslant C_{2}\left(T+\ln \frac{1}{\varepsilon}\right) \ln \frac{1}{\varepsilon}
$$

для $T \geqslant T_{0} u \varepsilon \in\left(0, \varepsilon_{0}\right)$.

Заметим, что левая часть оценки (4.17) точна при $T \geqslant \ln (1 / \varepsilon)$, но далека от оптимальной при $T \ll \ln (1 / \varepsilon)$. В частности, (4.17) не дает никакой информации об энтропии глобального аттрактора $\mathbb{A}$ на сечении. Следующая теорема дает оценку снизу для энтропии глобального аттрактора в случае, когда траекторный аттрактор удовлетворяет оценке (4.17).

ТЕОРема 4.5. Пусть выполнены условия теоремы 2.7, и пусть в-энтропия аттрактора $\mathscr{A}^{\mathrm{traj}}$ уравнения (0.4) удовлетворяет (4.17). Тогда существуют положительные константы $C$ и $\varepsilon_{0}$ такие, что энтропия глобального аттрактора $\mathbb{A}$, построенного в теореме 2.7 , допускает оценку

$$
\mathbf{H}_{\varepsilon}\left(\mathbb{A}, \mathbb{H}^{3 / 2}\right) \geqslant C\left(\ln \frac{1}{\varepsilon}\right)^{3 / 2} \quad \text { npu } \quad \varepsilon \in\left(0, \varepsilon_{0}\right)
$$

ДокАЗАТЕльство. Действительно, так как решения, принадлежашие $\mathscr{A}^{\text {traj }}$, paвномерно ограничены: $\|(u(t), \dot{u}(t))\|_{\mathbb{H}^{1}} \leqslant B_{*}$, то из оценки $(2.9)$ следует, что

$$
\begin{aligned}
& \left\|\left(u_{1}-u_{2}, \dot{u}_{1}-\dot{u}_{2}\right)\right\|_{C\left([0, t], \mathbb{H}^{1}\right)} \\
& \quad \leqslant\left\|\left(u_{1}(0)-u_{2}(0), \dot{u}_{1}(0)-\dot{u}_{2}(0)\right)\right\|_{\mathbb{H}^{1}}^{1-t / T} B_{*}^{t / T} e^{2 M(M+4 / T) t(T-t)}
\end{aligned}
$$

для любого $t<T$. Зафиксировав в этой оценке $T=2 t$ и предположив без ограничения обшности, что $t, M \geqslant 1$, получим

$$
\mathbf{H}_{\mu(\varepsilon)}\left(\mathscr{A}^{\text {traj }}, C\left([0, t], \mathbb{H}^{1}\right)\right) \leqslant \mathbf{H}_{\varepsilon}\left(\mathbb{A}, \mathbb{H}^{1}\right), \quad \text { где } \mu(\varepsilon)=\varepsilon^{1 / 2} B_{*}^{1 / 2} e^{20 M^{2} t^{2}}
$$

После замены $t$ на $T$ и применения оценки снизу (4.17) к левой части полученного неравенства мы получим неравенство

$$
\mathbf{H}_{\varepsilon}\left(\mathbb{A}, \mathbb{H}^{1}\right) \geqslant C_{1} T \ln \mu(\varepsilon)=C_{1} T\left(\frac{1}{2} \ln \frac{1}{\varepsilon}-\frac{1}{2} \ln B_{*}-20 M^{2} T^{2}\right)
$$

Максимизируя правую часть этого неравенства по $T$ (т.е. фиксировав $T:=(10 M)^{-1} \times$ $\left.(\ln (1 / \varepsilon))^{1 / 2}\right)$, мы выведем оценку (4.18). Теорема 4.5 доказана. 


\section{$\S 5$. Хаос в пространственных динамических системах}

В этом параграфе мы дадим интерпретацию результатов, полученных в предыдуших параграфах, в духе теории динамических систем. Для этого нам необходимо напомнить некоторые количественные характеристики динамической сложности, используемые в теории ДС. Мы начнем с классического понятия топологической энтропии (см., например, [19]).

ОПРЕДЕЛЕНИЕ 5.1. Пусть $(M, d)$ - компактное метрическое пространство, а $S_{h}: M \rightarrow M, h \in \mathbb{R}^{+},-$непрерьвная полугруппа, действуюшая в нем. Для любого $R>0$ определим новую метрику $d_{R}$ в $M$ следуюшим образом:

$$
d_{R}\left(m_{1}, m_{2}\right):=\sup _{h \leqslant R} d\left(S_{h} m_{1}, S_{h} m_{2}\right), \quad m_{1}, m_{2} \in M
$$

Тогда очевидно, что $\left(M, d_{R}\right)$ также является компактным метрическим пространством. Топологической энтропией полугруппы $S_{h}$ в $M$ назьвается число

$$
h_{\mathrm{top}}\left(S_{h}, M\right):=\lim _{\varepsilon \rightarrow 0} \limsup _{R \rightarrow \infty} \frac{1}{R} \mathbf{H}_{\varepsilon}\left(M, d_{R}\right),
$$

где символ $\mathbf{H}_{\varepsilon}\left(M, d_{R}\right)$ обозначает $\varepsilon$-энтропию множества $M$ в метрике (5.1).

Известно (см. [19]), что топологическая энтропия (5.2) не зависит от выбора метрики $d$ в $M$, а зависит только от топологии пространства $M$.

Для того чтобы применить общее определение 5.1 к исследованию траекторной ДС (2.1), необходимо фиксировать какую-нибудь метрику в $\mathscr{A}^{\text {traj }}$. Для этого нам понадобится следующее простое предложение.

ПРЕДЛОЖЕНИЕ 5.2. Пусть весовая функиия $\phi \in C_{\mathrm{bd}}(\mathbb{R})$ удовлетворяет условиям $\lim _{|t| \rightarrow \infty} \phi(t)=0 u \phi(t)>0$. Тогда топология, индуцированная в $\mathscr{A}^{\text {traј }}$ вложением $\mathscr{A}^{\text {traj }} \subset W_{\mathrm{bd}, \phi}^{2}\left(\mathbb{R}^{+}\right)$, совпадает с локальной топологией, индуцированной вложсением $\mathscr{A}^{\text {traj }} \subset W_{\mathrm{loc}}^{2}\left(\mathbb{R}_{+}\right)$.

Действительно, предложение 5.2 является немедленным следствием ограниченности множества $\mathscr{A}^{\text {traj }}$ в $W_{\text {bd }}^{2}\left(\mathbb{R}^{+}\right)$.

Зафиксируем теперь произвольную весовую функцию $\phi$, удовлетворяющую условиям предложения 5.2 , и определим метрику в $\mathscr{A}^{\text {traj }}$ по формуле

$$
d_{\phi}\left(u_{1}, u_{2}\right):=\left\|u_{1}-u_{2}\right\|_{W_{\mathrm{bd}, \phi}^{2}\left(\mathbb{R}^{+}\right)}, \quad u_{1}, u_{2} \in \mathscr{A}^{\text {traj }} .
$$

Тогда, согласно теореме 2.4 и предложению $5.2,\left(\mathscr{A}^{\text {traj }}, d_{\phi}\right)$ - компактное метрическое пространство, и можно определить топологическую энтропию $h_{\text {top }}\left(\mathscr{T}_{h}, \mathscr{A}^{\text {traj }}\right)$ траекторной ДС (2.1) по формуле (5.2). Следуюшее предложение дает более удобную формулу для ее вычисления.

ПреДЛОЖЕНИЕ 5.3. Топологическая энтропия $h_{\text {top }}\left(\mathscr{T}_{h}, \mathscr{A}^{\text {traj }}\right)$ не зависит от выбора весовой функции ф и вычисляется по следующей формуле:

$$
h_{\text {top }}\left(\mathscr{T}_{h}, \mathscr{A}^{\text {traj }}\right)=\lim _{\varepsilon \rightarrow 0} \limsup _{T \rightarrow \infty} \frac{1}{T} \mathbf{H}_{\varepsilon}\left(\left.\mathscr{A}^{\text {traj }}\right|_{(0, T)}, W_{\mathrm{bd}}^{2}(0, T)\right) .
$$


Доказательство формулы (5.4) более или менее очевидно и дано, например, в работе [39].

Заметим теперь, что, в отличие от классических ДС, порожденных ОДУ или большинством естественных УрЧП в ограниченных областях (см., например, [34]), в нашем случае топологическая энтропия может быть бесконечной. В частности, это так в случае системы (4.15).

СледСтВИЕ 5.4. Топологическая энтропия динамической системы $\left(\mathscr{T}_{h}, \mathscr{A}^{\text {traj}}\right)$, порожденной уравнением (4.15), бесконечна:

$$
h_{\text {top }}\left(\mathscr{T}_{h}, \mathscr{A}^{\text {traj }}\right)=\infty
$$

Действительно, (5.5) является немедленным следствием (5.4) и (4.17).

Напомним, что ДС с бесконечной топологической энтропией естественно возникают при изучении пространственной и динамической сложности аттракторов $\mathscr{A}$ glob эволюционных УрЧП в неограниченных областях, в частности, уравнений вида (0.13) (см., например, [39], [40]). Таким образом, вспомнив о вложении (0.14), естественно применить методы, разработанные там, для изучения траекторной ДС (2.1). Мы начнем с формулировки одного из возможных обобшений понятия топологической энтропии (см. [22], [39]).

Определение 5.5. Пусть $(M, d)$ - компактное метрическое пространство, а $S_{h}: M \rightarrow M-$ полугруппа в нем. Тогда модифицированная топологическая энтропия полугрупшы $S_{h}$ определяется следуюшим образом:

$$
\widehat{h}_{\mathrm{top}}\left(S_{h},(M, d)\right):=\limsup _{\varepsilon \rightarrow 0}\left(\ln \frac{1}{\varepsilon}\right)^{-1} \limsup _{R \rightarrow \infty} \frac{1}{R} \mathbf{H}_{\varepsilon}\left(M, d_{R}\right),
$$

где метрика $d_{R}$ определяется по формуле (5.1). Более того, следуя [22], введем среднюю топологическую размерность $\operatorname{dim}_{\text {top }}$ по формуле

$$
\operatorname{dim}_{\text {top }}\left(S_{h}, M\right):=\inf _{\widehat{d}} \widehat{h}_{\text {top }}\left(S_{h},(M, \widehat{d})\right),
$$

где нижняя грань берется по всем метрикам $\widehat{d}$ в $M$, которые порождают ту же самую топологию в $M$, что и метрика $d$.

В отличие от топологической энтропии, выражение (5.6) сохраняется только при липшицевых гомеоморфизмах (как и фрактальная размерность). Это и является причиной введения величины (5.7), которая так же, как и классическая топологическая энтропия, является топологическим инвариантом.

Итак, зафиксировав весовую функцию $\phi$, удовлетворяющую условиям предложения 5.2, и определив метрику $d_{\phi}$ в $\mathscr{A}^{\text {traj }}$ по формуле (5.3), можно определить модифицированную топологическую энтропию $\widehat{h}_{\text {top }}\left(\mathscr{T}_{h},\left(\mathscr{A}^{\text {traj }}, d_{\phi}\right)\right)$ и среднюю топологическую размерность $\operatorname{dim}_{\text {top }}\left(\mathscr{T}_{h}, \mathscr{A}^{\text {traj }}\right)$ траекторной ДС (2.1) по формулам (5.6) и (5.7). (Подчеркнем, что нижняя грань в (5.7) берется не только по метрикам вида (5.3), а по всем метрикам в $\mathscr{A}^{\text {traj }}$, задаюшим локальную топологию.)

Следуюшее утверждение является аналогом предложения 5.3. 
ПРЕДЛОЖЕНИЕ 5.6. Модифицированная топологическая энтропия траекторной динамической системы (2.1) не зависит от выбора веса ф и вычисляется по формуле

$$
\widehat{h}_{\text {top }}\left(\mathscr{T}_{h},\left(\mathscr{A}^{\text {traj }}, d_{\phi}\right)\right)=\limsup _{\varepsilon \rightarrow 0}\left(\ln \frac{1}{\varepsilon}\right)^{-1} \limsup _{T \rightarrow \infty} \frac{1}{T} \mathbf{H}_{\varepsilon}\left(\left.\mathscr{A}^{\text {traj }}\right|_{(0, T)}, W_{\text {bd }}^{2}(0, T)\right) .
$$

Доказательство (5.8) аналогично доказательству (5.4) (см. [39]).

Выражение в правой части (5.8) интерпретировано в работе [10] как (фрактальная) размерность аттрактора на единицу объема (см. (3.2)). Конечность и положительность этой величины для аттракторов $\mathscr{A}^{\text {glob }}$ большого класса эволюционных УрЧП в неограниченных областях доказана в [10], [39], [41], [42], [14]. Следующий результат показывает, что эта величина может быть строго положительной и в случае аттракторов уравнений вида (0.4).

СлЕДСТВИЕ 5.7. При выполнении условий теоремы 2.4 модифицированная топологическая әнтропия траекторной динамической системы (2.1) конечна:

$$
\widehat{h}_{\text {top }}\left(\mathscr{T}_{h}, \mathscr{A}^{\text {traj }}\right) \leqslant C<\infty .
$$

Более того, эта величина строго положительна для уравнения (4.15):

$$
0<C_{1} \leqslant \widehat{h}_{\text {top }}\left(\mathscr{T}_{h}, \mathscr{A}^{\text {traj }}\right) \leqslant C<\infty
$$

Действительно, оценка (5.9) немедленно следует из (5.8) и (3.5), а оценка (5.10) из (4.17).

Напомним, что в классической теории ДС для изучения хаотической динамики обычно используются гомеоморфные вложения схем Бернулли в исследуемую ДС (см. [19] и цитируемую там литературу). Заметим, однако, что классические схемы Бернулли с конечным числом символов имеют конечную топологическую энтропию и, следовательно, не могут рассматриваться как адекватная модель для исследования случая бесконечной энтропии. В этом случае естественно использовать схемы Бернулли с бесконечнылм числом символов.

ОПРЕДЕЛЕНИЕ 5.8. Пусть $\mathscr{M}:=[-1,1]^{\mathbb{Z}}$ - компактное метрическое пространство, наделенное тихоновской топологией. Напомним, что $\mathscr{M}$ состоит из функций $v: \mathbb{Z} \rightarrow$ $[-1,1]$, а топология в нем порождается стандартной метрикой:

$$
d\left(v_{1}, v_{2}\right):=\sum_{i=-\infty}^{\infty} 2^{-|i|}\left|v_{1}(i)-v_{2}(i)\right|
$$

Определим модельную ДС $\left(\mathscr{T}_{l}, \mathscr{M}\right)$ сдвигов в $\mathscr{M}$ по формуле

$$
\left(\mathscr{T}_{l} v\right)(i):=v(i+l), \quad i, l \in \mathbb{Z}, \quad v \in \mathscr{M}
$$

Известно, что $h_{\text {top }}\left(\mathscr{T}_{l}, \mathscr{M}\right)=\infty$ и $\widehat{h}_{\text {top }}\left(\mathscr{T}_{l},(\mathscr{M}, d)\right)=\operatorname{dim}_{\text {top }}\left(\mathscr{T}_{l}, \mathscr{M}\right)=1$.

Следуюшая теорема дает вложение ДС $\left(\mathscr{T}_{l}, \mathscr{M}\right)$ в траекторную ДС, порожденную уравнением (4.15). 
Теорема 5.9. Пусть $\mathscr{K}$ - существенное мноэсество уравнения (4.15). Тогда существует гомеоморфное вложение $\kappa: \mathscr{M} \rightarrow \mathscr{K}$ такое, что

$$
\mathscr{T}_{T l} \kappa(v)=\kappa\left(\mathscr{T}_{l} v\right), \quad l \in \mathbb{Z}, \quad v \in \mathscr{M},
$$

где $T>0$ - период, введенный в теореме 4.1 (и фиксированный как $T:=2 \pi$ в (4.13)). Более того, это вложсение липшицево в следующем смысле:

$$
\begin{aligned}
& C_{1} \sum_{i=-\infty}^{\infty} e^{-T|i|}\left|v_{1}(i)-v_{2}(i)\right| \\
& \quad \leqslant\left\|\kappa\left(v_{1}\right)-\kappa\left(v_{2}\right)\right\|_{W_{\mathrm{bd}, e}^{2}|t|}(\mathbb{R}) \leqslant C_{2} \sum_{i=-\infty}^{\infty} e^{-T|i|}\left|v_{1}(i)-v_{2}(i)\right| .
\end{aligned}
$$

ДокАЗАТЕЛЬСТво. По построению уравнения (4.15), множество $\mathbb{L}_{R}$, определенное в (4.4) и (4.8), содержится в $\mathscr{K}$ для некоторого $R>0$. Следовательно, достаточно построить вложение $\kappa: \mathscr{M} \rightarrow \mathbb{L}_{R}$. Мы утверждаем, что такое вложение задается формулой

$$
\kappa(v)(t):=R \sum_{i=-\infty}^{\infty} v(i) u(t-i T), \quad v \in \mathscr{M},
$$

где $u(t)$ - решение, построенное в теореме 4.1. Действительно, формула (5.13) немедленно следует из (5.15). Таким образом, остается проверить оценки (5.14). Аналогично (4.7) доказьвается, что

$$
\|\kappa(v)\|_{W^{2}(\tau)} \leqslant C \sum_{i=\infty}^{\infty}|v(i)| e^{-(\tau-i T)^{2} / 2} \text { при } \tau \in \mathbb{R} .
$$

Правая часть оценки (5.14) является немедленным следствием этого неравенства. Для проверки его левой части напомним, что, согласно теореме 4.1, первьй коэффициент Фурье $u_{1}(t)$ решения $u(t)$ имеет конечный носитель supp $u_{1} \subset[0, T]$. Поэтому

$$
\|\kappa(v)\|_{W^{2}(\tau)} \geqslant\left\|\left\langle\kappa(v), e_{1}\right\rangle\right\|_{W_{2}^{2}(\tau, \tau+1)}=|v([\tau / T])| \cdot\left\|u_{1}\right\|_{W_{2}^{2}(0,1)} \geqslant C|v([\tau / T])| .
$$

Левая часть неравенства (5.14) является элементарным следствием этой оценки. Теорема 5.9 доказана.

Напомним, что уравнение (4.15) удовлетворяет условиям теоремы 2.7 , поэтому траекторная ДС (2.1) топологически сопряжена с ДС $\left(\mathbb{S}_{h}, \mathbb{K}\right)$ на сечении, определенной по формуле (2.16). Следуюший результат является аналогом теоремы 5.9 для этой ДС.

СЛЕДСТВИЕ 5.10. Пусть $\mathbb{A}=\Pi_{0} \mathscr{A}^{\text {traj }}$ - глобальный аттрактор уравнения (4.15). Тогда существует гомеоморфное вложсение $\widehat{\kappa}: \mathscr{M} \rightarrow \mathbb{A}$ такое, что

$$
\mathbb{S}_{T l} \widehat{\kappa}(v)=\widehat{\kappa}\left(\mathscr{T}_{l} v\right) \quad \text { для } л ю б ы x \quad l \in \mathbb{Z} \quad u \quad v \in \mathscr{M}
$$

Более того, этот гомеоморфизм сохраняют величины (5.6) и (5.7), т.е.

$$
\begin{aligned}
\widehat{h}_{\text {top }}\left(\mathbb{S}_{h}, \widehat{\kappa}(\mathscr{M})\right) & =T^{-1} \widehat{h}_{\mathrm{top}}\left(\mathscr{T}_{l}, \mathscr{M}\right), \\
\operatorname{dim}_{\text {top }}\left(\mathbb{S}_{h}, \widehat{\kappa}(\mathscr{M})\right) & =T^{-1} \operatorname{dim}_{\text {top }}\left(\mathscr{T}_{l}, \mathscr{M}\right) .
\end{aligned}
$$


ДокАЗАТЕльство. Согласно следствию 2.6 , оператор следа $\Pi_{0}: \mathscr{K} \rightarrow \mathbb{A}$ задает гёльдеров гомеоморфизм, константа Гёльдера которого сколь угодно близка к единице, при соответствуюшем выборе весовой функции в $\mathscr{K}$ (см. (2.21) и (2.22)). Определим гомеоморфизм $\widehat{\kappa}:=\Pi_{0} \circ \kappa$. Действительно, (5.16) является немедленным следствием (5.13). Так как средняя топологическая размерность является топологическим инвариантом, то второе равенство в (5.17) очевидно. Множитель $T^{-1}$ возникает из-за перенормировки "времени" (полугруппа $\left(\mathscr{T}_{l}, \mathscr{M}\right)$ сопряжена с $\left(\mathscr{T}_{T l}, \mathscr{K}\right)$ посредством $\kappa)$. Для того чтобы проверить первое равенство в (5.17), напомним, что $\kappa$ липшицево в смысле (5.14). Поэтому из инвариантности модифицированной топологической энтропии при липшицевых гомеоморфизмах и того факта, что (5.6) не зависит от выбора весовой функции $\phi$, удовлетворяющей условиям предложения 5.2, в метрике (5.3) следует, что

$$
\widehat{h}_{\text {top }}\left(\mathscr{T}_{h}, \kappa(\mathscr{M})\right)=T^{-1} \widehat{h}_{\text {top }}\left(\mathscr{T}_{l}, \mathscr{M}\right) .
$$

Аналогично, так как П гёльдеров с константой Гёльдера, сколь угодно близкой к единице, то $\widehat{h}_{\text {top }}\left(\mathscr{T}_{h}, \kappa(\mathscr{M})\right)=\widehat{h}_{\text {top }}\left(\mathbb{S}_{h}, \widehat{\kappa}(\mathscr{M})\right)$. Следствие 5.10 доказано.

СледСтвие 5.11. Средняя топологическая размерность динамической системы, порожденной уравнением (4.15), строго положительна:

$$
\operatorname{dim}_{\text {top }}\left(\mathscr{T}_{h}, \mathscr{A}^{\text {traj }}\right)=\operatorname{dim}_{\text {top }}\left(\mathbb{S}_{h}, \mathbb{A}\right) \geqslant T^{-1}>0
$$

Действительно, как известно (см. [22]), $\operatorname{dim}_{\text {top }}\left(\mathscr{T}_{l}, \mathscr{M}\right)=1$. Поэтому оценка (5.19) является следствием (5.17).

Следующий результат показывает, что любую конечномерную динамику можно реализовать с точностью до гомеоморфизма, ограничивая $\mathbb{S}_{h}$ на соответствующее инвариантное множество аттрактора $\mathbb{A}$ уравнения (4.15).

СлЕДСТВИЕ 5.12. Пусть $\mathbb{A}$ - глобальный аттрактор уравнения (4.15), $K \subset \mathbb{R}^{N}$ - произвольный компакт и $F: K \rightarrow K$ - произвольный гомеоморфизм. Тогда существует гомеоморфное вложение $\tau: K \rightarrow \mathbb{A}$ такое, ито

$$
\mathbb{S}_{N T} \circ \tau(k)=\tau(F k) \quad \text { для } \text { любого } \quad k \in K .
$$

ДоКАЗАТЕЛЬСтво. Действительно, благодаря следствию 5.10, достаточно вложить ДС $(F, K)$ в $\left(\mathscr{T}_{l}, \mathscr{M}\right)$. Более того, без ограничения общности можно предположить, что $K \subset[-1,1]^{N}$. Тогда необходимое вложение задается формулой

$$
\widetilde{\tau}(k)(i):=\left(F^{(m)}(k)\right)_{j}, \quad i=m N+j, \quad m, j \in \mathbb{Z}, \quad 0 \leqslant j \leqslant N-1,
$$

где через $F^{(m)}$ обозначена $m$-я итерация отображения $F,(k)_{j}-j$-я координата точки $k \in[-1,1]^{N}$. Тогда отображение $\tau:=\widehat{\kappa} \circ \widetilde{\tau}$, очевидно, удовлетворяет всем условиям следствия. 
ЗАмЕчАниЕ 5.13. Напомним, что абстрактное эллиптическое уравнение (0.4) может быть формально интерпретировано как эволющионное уравнение второго порядка. Более того, эта интерпретация частично оправдьвается (при вьполнении условий теоремы 2.7) построением по уравнению (0.4) непрерывной ДС $\left(\mathbb{S}_{h}, \mathbb{K}\right)$. Но, в отличие от случая эволющионных уравнений, ДС, порождаемые эллиптическими уравнениями, не являются липшицевыми в обшем случае (мы не можем взять $\alpha=0$ в (2.18)), а только гёльдеровы с константой Гёльдера, сколь угодно близкой к единице (как в случае уравнения (4.15)). Отсутствие липшицевости позволяет таким системам иметь бесконечномерные аттракторы с бесконечной топологической энтропией. Более того, можно показать, рассуждая стандартным образом, что если некоторое уравнение, удовлетворяюшее условиям теоремы 2.7 , допускает также свойство липшицевости в виде

$$
\left\|\mathbb{S}_{h}\left(z_{1}\right)-\mathbb{S}_{h}\left(z_{2}\right)\right\|_{\mathbb{H}^{1}} \leqslant Q_{h}\left(\left\|z_{1}\right\|_{\mathbb{H}^{3 / 2}}+\left\|z_{2}\right\|_{\mathbb{H}^{3 / 2}}\right)\left\|z_{1}-z_{2}\right\|_{\mathbb{H}^{1}}, \quad z_{i} \in \mathbb{K}
$$

то его глобальный аттрактор имеет конечную фрактальную размерность, а значит, его топологическая энтропия также конечна (как и в случае эволющионных уравнений).

ЗАмечАнИЕ 5.14. Заметим, что, как и в случае классических ДС, наше вложение схемы Бернулли в траекторную ДС, порожденную уравнением (4.15), основано на нахождении подходящей гомоклинической орбиты. Действительно, решение $t \mapsto$ $(\sin t, \cos t, u(t))$, где $u(t)$ - функция, построенная в теореме 4.1, является гомоклинической орбитой по отношению к $2 \pi$-периодическому решению $t \mapsto(\sin t, \cos t, 0)$. Но, в отличие от классического случая, в нашей ситуации мы можем суммировать пространственные сдвиги исходной гомоклинической орбитыне только с коэффициентами из $\{0,1\}$, но из интервала $[-1,1]$ (см. (5.15)). Более того, теорема 5.9 показывает, что периодическая орбита $(\sin t, \cos t, 0)$ является "бесконечно вырожденной". Действительно, в ее окрестности имеется однопараметрическое семейство $2 \pi$-периодических орбит, которое параметризуется константами $v_{\varepsilon} \in \mathscr{M}, \varepsilon \in[-1,1]$ (т.е. $v_{\varepsilon}(i) \equiv \varepsilon$ при $i \in \mathbb{Z})$. Аналогично, имеется двупараметрическое семейство $4 \pi$-периодических решений, трехпараметрическое семейство $6 \pi$-периодических решений и т. д. Замыкание этого огромного количества периодических орбит и дает вложение схемы Бернулли $\left(\mathscr{T}_{l}, \mathscr{M}\right)$, построенное в теореме 5.9 .

ЗАмЕчАНИЕ 5.15. Гомеоморфные вложения модельной ДС $\left(\mathscr{T}_{l}, \mathscr{M}\right)$ в пространственную ДС $\left(\mathscr{T}_{h}, \mathscr{A}^{\text {glob }}\right)$, действующую на аттракторе, были построены в работах [39], [40] для весьма широкого класса систем уравнений реакции-диффузии вида (0.13). Более того, из предложенной там конструкции следует, что образ $\mathscr{M}$ лежит в сильно неустойчивом многообразии (по отношению к эволюционной переменной) некоторого пространственно-однородного положения равновесия рассматриваемого эволюционного уравнения. Теорема 5.9 показьвает, что для некоторых эволюционных уравнений в неограниченных областях пространственно-хаотическое поведение этого типа может быть обнаружено и на множестве положений $\mathscr{K}$ равновесия, которое априори существенно меньше, чем $\mathscr{A}^{\text {glob }}$.

\section{СПИСОК ЛИТЕРАТУРЫ}

[1] S. Agmon, L. Nirenberg. Lower bounds and uniqueness theorems for solutions of differential equations in a Hilbert space // Comm. Pure Appl. Math. 1967. V. 20. P. 207-229. 
[2] А. В. Бабин. Аттрактор обобщенной полугруппы, порожденной эллиптическим уравнением в цилиндрической области // Изв. РАН. Сер. матем. 1994. Т. 58. № 2. С. 3-18.

[3] A. V. Babin. Inertial manifolds for traveling-wave solutions of reaction-diffusion systems // Comm. Pure Appl. Math. 1995. V. 48. № 2. P. 167-198.

[4] А. В. Бабин, М.И. Вишик. Аттракторы эволюционных уравнений. М.: Наука, 1989.

[5] T. J. Bridges, A. Mielke. Instability of spatially-periodic states for a family of semilinear PDE's on an infinite strip // Math. Nachr. 1996. V. 179. P. 5-25.

[6] À. Calsina, X. Mora, J. Solà-Morales. The dynamical approach to elliptic problems in cylindrical domains, and a study of their parabolic singular limit // J. Differential Equations. 1993. V. 102. № 2. P. 244-304.

[7] À. Calsina, J. Solà-Morales, M. València. Bounded solutions of some nonlinear elliptic equations in cylindrical domains // J. Dynam. Differential Equations. 1997. V. 9. № 3. P. 343-372.

[8] V. V. Chepyzhov, M. I. Vishik. Evolution equations and their trajectory attractors // J. Math. Pures Appl. (9). 1997. V. 76. № 10. P. 913-964.

[9] В.В. Чепыжов, М.И. Вишик. Колмогоровская $\varepsilon$-энтропия аттракторов систем реакции-диффузии // Матем. сб. 1998. Т. 189. № 2. С. 235-263.

[10] P. Collet, J.-P. Eckmann. Extensive properties of the complex Ginzburg-Landau equation // Comm. Math. Phys. 1999. V. 200. № 3. P. 699-722.

[11] P. Collet, J.-P. Eckmann. Topological entropy and $\varepsilon$-entropy for damped hyperbolic equations // Ann. Henri Poincaré. 2000. V. 1. P. 715-752.

[12] G. Dangelmayr, B. Fiedler, K. Kirchgässner, A. Mielke. Dynamics of Nonlinear Waves in Dissipative Systems: Reduction, Bifurcation and Stability. Harlow: Longman, 1996. (Pitman Res. Notes Math. Ser. V. 352.)

[13] M. A. Efendiev, S. V. Zelik. The attractor for a nonlinear reaction-diffusion system in an unbounded domain // Comm. Pure Appl. Math. 2001. V. 54. № 6. P. 625-688.

[14] M. A. Efendiev, S. V. Zelik. Upper and lower bounds for the Kolmogorov entropy of the attractor for an RDE in an unbounded domain // J. Dynam. Differential Equations. 2002. V. 14. № 2. P. 369-403.

[15] B. Fiedler, A. Scheel, M. I. Vishik. Large patterns of elliptic systems in infinite cylinders // J. Math. Pures Appl. (9). 1998. V. 77. № 9. P. 879-907.

[16] M. D. Groves, J. F. Toland. On variational formulations for steady water waves // Arch. Rational Mech. Anal. 1997. V. 137. № 3. P. 203-226.

[17] G. Iooss, K. Kirchgässner. Water waves for small surface tension: an approach via normal form // Proc. Roy. Soc. Edinburgh Sect. A. 1992. V. 122. P. 267-299.

[18] G. Iooss, A. Mielke. Bifurcating time-periodic solutions of Navier-Stokes equations in infinite cylinders // J. Nonlinear Sci. 1991. V. 1. № 1. P. 107-146.

[19] A. Katok, B. Hasselblatt. Introduction to the Modern Theory of Dynamical Systems. Cambridge: Cambridge Univ. Press, 1995.

[20] K. Kirchgässner. Wave-solutions of reversible systems and applications // J. Differential Equations. 1982. V. 45. P. 113-127.

[21] А. Н. Колмогоров, В. М. Тихомиров. $\varepsilon$-энтропия и $\varepsilon$-емкость множеств в функциональных пространствах // УМН. 1959. Т. 14. № 2. С. 3-86.

[22] E. Lindenstraus, B. Weiss. Mean topological dimension // Israel J. Math. 2000. V. 115. P. 1-24.

[23] A. Mielke. Reduction of quasilinear elliptic equations in cylindrical domains with applications // Math. Methods Appl. Sci. 1988. V. 10. № 1. P. 51-66.

[24] A. Mielke. Normal hyperbolicity of center manifolds and Saint-Venant's principle // Arch. Rational Mech. Anal. 1990. V. 110. № 4. P. 353-372.

[25] A. Mielke. Hamiltonian and Lagrangian Flows on Center Manifolds. With applications to elliptic variational problems. Berlin: Springer-Verlag, 1991. (Lecture Notes in Math. V. 1489.)

[26] A. Mielke. On nonlinear problems of mixed type: a qualitative theory using infinite-dimensional center manifolds // J. Dynam. Differential Equations. 1992. V. 4. P. 419-443.

[27] A. Mielke. Floquet theory for, and bifurcations from spatially periodic patterns // Tatra Mt. Math. Publ. 1994. V. 4. P. 153-158. 
[28] A. Mielke. Essential manifolds for an elliptic problem in an infinite strip // J. Differential Equations. 1994. V. 110. № 2. P. 322-355.

[29] A. Mielke. The complex Ginzburg-Landau equation on large and unbounded domains: sharper bounds and attractors // Nonlinearity. 1997. V. 10. № 1. P. 199-222.

[30] A. Mielke. Exponentially weighted $L^{\infty}$-estimates and attractors for parabolic systems on unbounded domains // International Conference on Differential Equations (EQUADIFF 99), Berlin, 1999 / ed. B. Fiedler, K. Gröger, and J. Sprekels. Singapore: World Scientific, 2000. P. 641-646.

[31] A. Mielke, G. Schneider. Attractors for modulation equations on unbounded domains existence and comparison // Nonlinearity. 1995. V. 8. № 5. P. 743-768.

[32] D. Peterhof, B. Sandstede, A. Scheel. Exponential dichotomies for solitary-wave solutions of semilinear elliptic equations on infinite cylinders // J. Differential Equations. 1997. V. 140. № 2. P. 266-308.

[33] B.-W. Schulze, M.I. Vishik, I. Witt, S. V. Zelik. The trajectory attractor for a nonlinear elliptic system in a cylindrical domain with piecewise smooth boundary // Rend. Accad. Naz. Sci. XL Mem. Mat. Appl. (5). 1999. V. 23. P. 125-166.

[34] R. Temam. Infinite-Dimensional Dynamical Systems in Mechanics and Physics. New York: Springer-Verlag, 1988.

[35] H. Triebel. Interpolation Theory, Function Spaces, Differential Operators. Amsterdam: North-Holland, 1978.

[36] М. И. Вишик, С. В. Зелик. Траекторный аттрактор нелинейной эллиптической системы в цилиндрической области // Матем. сб. 1996. Т. 187. № 12. С. 21-56.

[37] М. И. Вишик, С.В.Зелик. Регулярньй аттрактор нелинейной эллиптической системы в цилиндрической области // Матем. сб. 1999. Т. 190. № 6. С. 23-58.

[38] С.В. Зелик. Аттрактор нелинейной системы реакции-диффузии в $\mathbb{R}^{n}$ и оценки его $\varepsilon$-энтропии // Матем. заметки. 1999. Т. 65. №6. С. 941-944.

[39] S. V. Zelik. The attractors of reaction-diffusion systems in unbounded domains and their spatial complexity // Preprint 32/00. Berlin: DANSE, 2000.

[40] S. V. Zelik. Spatial and dynamical chaos generated by reaction diffusion systems in unbounded domains // Preprint 38/00. Berlin: DANSE, 2000.

[41] S. V. Zelik. The attractor for a nonlinear hyperbolic equation in an unbounded domain // Discrete Contin. Dynam. Systems. 2001. V. 7. № 3. P. 593-641.

[42] S. V. Zelik. The attractor for a nonlinear reaction-diffusion system in an unbounded domain and Kolmogorov's $\varepsilon$-entropy // Math. Nachr. 2001. V. 232. № 1. P. 129-179.

Universität Stuttgart, Germany;

Поступила в редакцию

Институт проблем передачи информации РАН, Москва

05.04 .2002 